\title{
Trifluoroacetylation of Alcohols During NMR Study of Compounds with Bicyclo[2.2.1]heptane, Oxabicyclo[3.3.0]octane and Bicyclo[3.3.0]octane Skeleton
}

\author{
CONSTANTIN I. TANASE ${ }^{1 *}$, ANAMARIA HANGANU ${ }^{2}$, CONSTANTIN DRAGHICI $^{2}$ \\ ${ }^{1}$ National Institute for Chemical-Pharmaceutical Research and Development, Department of Bioactive Substances and \\ Pharmaceutical Technologies, 112 Vitan Av., 031299 Bucharest, Romania \\ ${ }^{2}$ Organic Chemistry Center C.D.Nenitescu, 202B Splaiul Independentei, 060023, Bucharest, Romania
}

\begin{abstract}
TFA was added to a solution of a bicyclo[2.2.1] heptane azide-alcohol in $\mathrm{CDCl}_{3}$ to correctly characterize the compound, but during 24 h gave the trifluoro acetylated compound in quantitative yield. NMR spectra of the esterified compound helped us also to correctly attribute the NMR signals to the protons, and also confirmed the identification of the carbon atoms. The study was extended to other 14 compounds containing a primary alcohol group alone or with an ethylene ketal, a $\delta$ - or $\gamma$-lactone group, a primary and a secondary group, two primary and an alkene group and two primary and a secondary alcohol groups on scaffolds containing bicyclo[2.2.1]heptane, oxabicyclo[3.3.0]octane, bicyclo [2.2.1]heptane constrained with a cyclopropane ring and bicyclo[3.3.0]octane fragments. The esterification of all compounds was also quantitative in 24 to $72 \mathrm{~h}$; this helped us to correct attribute the NMR signals to the protons and carbon atoms of the un-esterified compounds by comparison with those of the trifluoro acetylated compounds. A graphical presentation of ${ }^{1} \mathrm{H}$ - and ${ }^{13} \mathrm{C}-\mathrm{NMR}$ spectra of a few un-esterified and esterified compounds are presented in the paper.
\end{abstract}

Keywords: $\mathrm{CF}_{3} \mathrm{COOH}$, alcohols esterification, $\mathrm{NMR}$ tube trifluoroacetylation, bicyclo[2.2.1] heptane alcohols, oxabicyclo[3.3.0]octane, Corey lactol, bicyclo[3.3.0]octane, prostaglandin enone, prostaglandin diols

\section{Introduction}

A routine NMR characterization of a compound with a primary alcohol group needed to distinguish between two protons and after adding trifluoroacetic acid (TFA) in the tube we observed the appearance of signals which proved to be those of the trifluoro acetylated compound formed quantitatively after a day. Browsing the literature, we found that alcohols are even quantitative esterified with TFA [1, 2]. For example, synthesis of ethyl trifluoroacetate from ethyl alcohol and TFA is also mentioned in patents [36] and journals [7]. Methyl and isopropyl trifluoroacetate was also synthesized from TFA and methanol or isopropanol $[8,9]$. The esterification of alcohols in TFA as solvent was performed and followed in NMR tube [10-12] to observe the transformation of alcohols into the corresponding trifluoroacetates, because the deshielding effect of the trifluoro acetyl group produces a considerable shift of the signal for the proton(s) linked to the hydroxyl group, by comparison with that of the corresponding protons in trifluoroacetate compound, which are moved to lower field. Though an esterification of hydroxyl groups were observed in $\mathrm{CDCl}_{3}+\mathrm{TFA}$ during NMR spectra registration, a paper on the trifluoroacetyl esterification during NMR studies was not found in the literature. And the paper is presented as a scholar theme exemplified for primary and secondary alcohol groups linked to different bicyclic and tricyclic scaffolds and in different configurations.

\section{Materials and methods}

The compounds were dissolved in $\mathrm{CDCl}_{3}$, and ${ }^{1} \mathrm{H},{ }^{13} \mathrm{C}, 2 \mathrm{D}-\mathrm{NMR}$ spectra have been done. Then near $0.03 \mathrm{~mL}$ TFA were added and the NMR spectra were followed until the esterification approach the end in specified time of reaction. The same spectra have been done in DMSO- $\mathrm{d}_{6}$ for a few of the compounds.

\footnotetext{
*email:cvtanase@gmail.com
} 
${ }^{1} \mathrm{H}$ - and ${ }^{13} \mathrm{C}-\mathrm{NMR}$ spectra were recorded on Bruker spectrometer $\left(500 \mathrm{MHz}\right.$ for ${ }^{1} \mathrm{H}$ and $125 \mathrm{MHz}$ for ${ }^{13} \mathrm{C}$ ), chemical shifts $(\delta)$ are given in ppm relative to TMS as internal standard. Complementary 2DNMR spectra were done for correct assignment of NMR signals. The numbering of the atoms in the compounds is presented in Figure 1.

1.NMR spectra of the compound $\mathbf{1} \boldsymbol{a}$ and of the trifluoro acetylated compound $\mathbf{1 b}$.

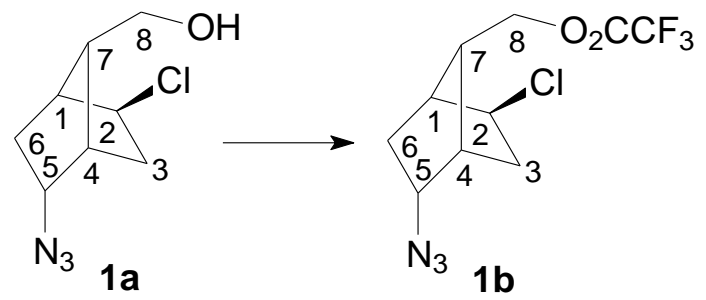

Compound 1a: ${ }^{1} \mathrm{H}-\mathrm{NMR}-500 \mathrm{MHz}\left(\mathrm{CDCl}_{3}, \delta \mathrm{ppm}, J \mathrm{~Hz}\right): 3.95(\mathrm{dd}, 1 \mathrm{H}, \mathrm{H}-8,7.6,11.4), 3.93-3.92$ (m, 2H, H-2, H-5), 3.92 (dd, 1H, H-8, 8.4, 11.4), 2.62 (dd, 1H, H-3, 8.0, 14.8), 2.51 (t, 1H, H-4, 4.3), 2.49 (d, 1H, H-1, 5.0), 2.13 (ddd, 1H, H-6, 5.0, 10.6, 13.8), 2.02 (t, 1H, H-7, 7.8), 2.00 (brt, 1H, H-3, 3.6, 14.8), 1.75 (s, $1 \mathrm{H}, \mathrm{OH}), 1.05$ (dd, $1 \mathrm{H}, \mathrm{H}-6,3.6,13.8),{ }^{13} \mathrm{C}-\mathrm{NMR}-125 \mathrm{MHz}\left(\mathrm{CDCl}_{3}, \delta \mathrm{ppm}, J \mathrm{~Hz}\right)$ : 60.53 (C-5), 60.35 (C-8), 59.91 (C-2), 51.74 (C-7), 47.03 (C-1), 43.10 (C-4), 36.27 (C-6), 34.05 (C-3).

Compound $1 \mathrm{~b}$, formed after one day with TFA: ${ }^{1} \mathrm{H}-\mathrm{NMR}-500 \mathrm{MHz}\left(\mathrm{CDCl}_{3}+\mathrm{TFA}, \delta \mathrm{ppm}, J \mathrm{~Hz}\right)$ : 4.78 (dd, 1H, H-8, 9.3, 11.4), 4.68 (dd, 1H, H-8, 6.2, 11.4), 3.99 (m, 1H, H-5) from COSY, 3.95 (dd, 1H, H-2, 3.8, 8.0), 2.71 (dd, 1H, H-3, 8.0, 15.2), 2.60 (t, 1H, H-4, 4.2), 2.56 (d, 1H, H-1, 4.8), 2.20 (ddd, 1H, H-6, 5.0, 10.7, 14.0), 2.19 (m, 1H, H-7), 2.06 (brt, 1H, H-3, 3.5, 15.2), 1.11 (dd, 1H, H-6, 3.6, 14.0), ${ }^{13} \mathrm{C}-\mathrm{NMR}-125 \mathrm{MHz}\left(\mathrm{CDCl}_{3}+\mathrm{TFA}, \delta \mathrm{ppm}, J \mathrm{~Hz}\right): 158.21\left(\mathrm{q}, \mathrm{COCF}_{3}, J=42.5\right), 114.55\left(\mathrm{q}, \mathrm{COCF}_{3}, J=\right.$ 283.8), 66.37 (C-8), 60.33 (C-5), 58.96 (C-2), 47.88 (C-7), 47.68 (C-1), 43.52 (C-4), 35.82 (C-6), 33.85 (C-3).

2.NMR spectra of the compound $\mathbf{2 a}$ and of the trifluoroacetylated compound $\mathbf{2 b}$.

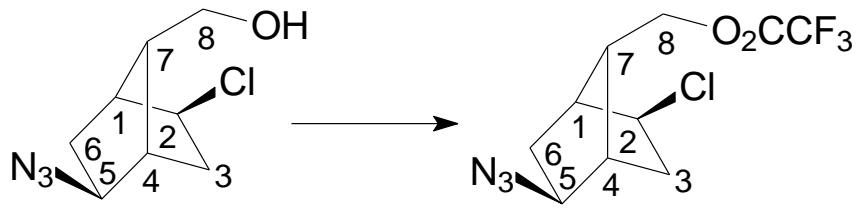

2a

$2 b$

Compound 2a: ${ }^{1} \mathrm{H}-\mathrm{NMR}-500 \mathrm{MHz}\left(\mathrm{CDCl}_{3}, \delta\right.$ ppm, $\left.J \mathrm{~Hz}\right): 3.98$ (dd, $\left.1 \mathrm{H}, \mathrm{H}-8,7.9,11.4\right), 3.91$ (dd, 1H, H-8, 8.2 11.4), 3.94 (dd, 1H, H-2, 3.8, 7.9), 3.49 (dd, 1H, H-5, 3.3, 7.6), 2.54 (d, 1H, H-1, 4.6), 2.40 (d, 1H, H-4, 4.5), 2.25 (t, 1H, H-7, 8.0), 2.14 (dd, 1H, H-3, 4.3, 15.0), 2.01 (dd, 1H, H-3, 8.0, 15.0), 1.69 (dd, 1H, H-6, 7.7, 14.0), 1.66 (s, 1H, OH), 1.59 (dt, 1H, H-6, 4.0, 14.0), ${ }^{13} \mathrm{C}-\mathrm{NMR}-125 \mathrm{MHz}\left(\mathrm{CDCl}_{3}, \delta\right.$ ppm, $J$ Hz): 60.88 (C-5), 60.34 (C-8), 59.45 (C-2), 49.65 (C-7), 46.14 (C-1), 43.99 (C-4), 37.69 (C-6), 37.21 (C-3), (NMR spectra in $\left.\mathrm{CDCl}_{3},[13-15]\right)$.

Compound $2 \mathrm{~b}$, formed after one day with TFA: ${ }^{1} \mathrm{H}-\mathrm{NMR}-500 \mathrm{MHz}\left(\mathrm{CDCl}_{3}+\mathrm{TFA}, \delta \mathrm{ppm}, J \mathrm{~Hz}\right)$ : 4.75 (dd, 1H, H-8, 8.8, 11.6), 4.73 (dd, 1H, H-8, 7.0, 11.6), 3.87 (dd, 1H, H-2, 4.0, 7.8) from COSY, 3.59 (dd, 1H, H-5, 3.2, 7.7), 2.61 (d, 1H, H-1, 4.4), 2.45 (d, 1H, H-4, 4.2), 2.40 (t, 1H, H-7, 7.9), 2.19 (dd, 1H, H-3, 4.3, 15.2), 2.11 (dd, 1H, H-3, 7.8, 15.2), 1.76 (dd, 1H, H-6, 7.7, 14.1), 1.65 (dt, 1H, H-6, $3.8,14.1),{ }^{13} \mathrm{C}-\mathrm{NMR}-125 \mathrm{MHz}\left(\mathrm{CDCl}_{3}+\mathrm{TFA}, \delta \mathrm{ppm}, J \mathrm{~Hz}\right): 158.21$ (q, $\left.\mathrm{COCF}_{3}, J=42.5\right), 114.55(\mathrm{q}$, $\left.\mathrm{COCF}_{3}, J=283.8\right), 66.25(\mathrm{C}-8), 62.49(\mathrm{C}-5), 58.53(\mathrm{C}-2), 46.90(\mathrm{C}-1), 45.67$ (C-7), 44.38 (C-4), 37.43 (C-6), 36.96 (C-3). 
3.NMR spectra of the compound $\mathbf{3 a}$ and of the trifluoroacetylated compound $\mathbf{3 b}$.

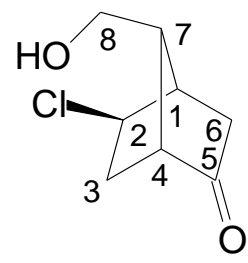

$3 a$

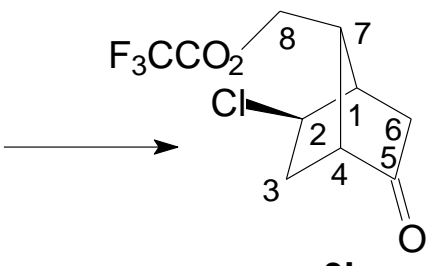

$3 \mathbf{b}$

Comppound 3a: ${ }^{1} \mathrm{H}-\mathrm{NMR}-500 \mathrm{MHz}\left(\mathrm{CDCl}_{3}, \delta \mathrm{ppm}, J \mathrm{~Hz}\right): 4.05(\mathrm{t}, 1 \mathrm{H}, \mathrm{H}-2,6.2), 3.95(\mathrm{~d}, 2 \mathrm{H}, \mathrm{H}-$ 8, 7.6), 2.86 (d, 1H, H-1, 4.8), 2.69 (s, 1H, H-4), 2.34 (s, 1H, OH), 2.23-2.28 (m, 3H, H-7, 2H-3), 2.23 (dd, $1 \mathrm{H}, \mathrm{H}-6,5.0,18.2), 1.91$ (d, $1 \mathrm{H}, \mathrm{H}-6,18.2),{ }^{13} \mathrm{C}-\mathrm{NMR}-125 \mathrm{MHz}\left(\mathrm{CDCl}_{3}, \delta \mathrm{ppm}, J \mathrm{~Hz}\right): 213.21(\mathrm{C}-$ 5), 59.62 (C-8), 57.78 (C-2), 52.16 (C-4), 51.05 (C-7), 46.20 (C-1), 45.40 (C-6), 34.21 (C-3).

Compound 3b, formed after 3 days (over weekend) with TFA: ${ }^{1} \mathrm{H}-\mathrm{NMR}-500 \mathrm{MHz}\left(\mathrm{CDCl}_{3}+\right.$ TFA, $\delta$ ppm, $J$ Hz): 4.79 (dd, 1H, H-8, 9.3, 11.6), 4.70 (dd, 1H, H-8, 6.1, 11.6), 4.10 (dd, 1H, H-2, 4.6, 7.2), 2.96 (d, 1H, H-1, 4.6), 2.84 (brd, 1H, H-4, 2.7), 2.54 (brdd, 1H, H-7, 6.1, 9.3), 2.42-2.37 (m, 2H, H-3), 2.35 (dd, 1H, H-6, 5.0, 18.6), 2.07 (d, 1H, H-6, 18.6), ${ }^{13} \mathrm{C}-\mathrm{NMR}-125 \mathrm{MHz}\left(\mathrm{CDCl}_{3}+\mathrm{TFA}, \delta\right.$ ppm, $J \mathrm{~Hz}): 216.30(\mathrm{C}-5), 157.47\left(\mathrm{q}, \mathrm{COCF}_{3}, J=42.5\right), 114.43\left(\mathrm{q}, \mathrm{COCF}_{3}, J=283.8\right), 64.95(\mathrm{C}-8), 56.62(\mathrm{C}-$ 2), 52.42 (C-4), 47.07 (C-7), 46.86 (C-1), 45.08 (C-6), 33.93 (C-3).

4.NMR spectra of the enantiomer compound $\mathbf{4 a}$ and of the trifluoroacetylated compound $\mathbf{4 b}$.

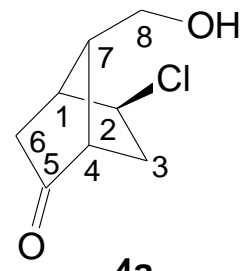

4a

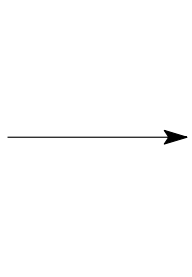

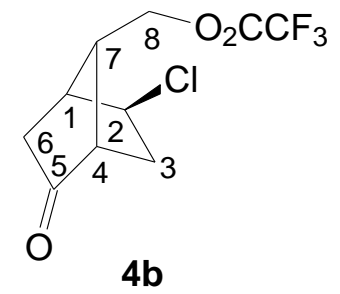

4b

Compound 4a: ${ }^{1} \mathrm{H}-\mathrm{NMR}-500 \mathrm{MHz}\left(\mathrm{CDCl}_{3}, \delta\right.$ ppm, $\left.J \mathrm{~Hz}\right): 4.03(\mathrm{t}, 1 \mathrm{H}, \mathrm{H}-2,6.2), 3.93$ (d, 2H, H-8, 7.7), 2.84 (d, 1H, H-1, 4.7), 2.68 (s, 1H, H-4), 2.57 (s, 1H, OH), 2.29 (t, 1H, H-7, 9.0), 2.29-2.26 (m, $2 \mathrm{H}, 2 \mathrm{H}-3), 2.21$ (dd, $1 \mathrm{H}, \mathrm{H}-6,4.9,18.2), 1.99$ (d, 1H, H-6, 18.2), ${ }^{13} \mathrm{C}-\mathrm{NMR}-125 \mathrm{MHz}\left(\mathrm{CDCl}_{3}, \delta \mathrm{ppm}, J\right.$ Hz): 213.31 (C-5), 59.53 (C-8), 57.74 (C-2), 52.14 (C-4), 51.04 (C-7), 46.17 (C-1), 45.37 (C-6), 34.14 (C-3). (See NMR spectra in DMSO, [16]).

Compound $4 \mathrm{~b}$, formed after $24 \mathrm{~h}$ with TFA: ${ }^{1} \mathrm{H}-\mathrm{NMR}-500 \mathrm{MHz}\left(\mathrm{CDCl}_{3}+\mathrm{TFA}, \delta \mathrm{ppm}, J \mathrm{~Hz}\right)$ : 4.79 (dd, 1H, H-8, 9.4, 11.5), 4.69 (dd, 1H, H-8, 6.1, 11.5), 4.10 (dd, 1H, H-2, 4.7, 7.1), 2.95 (d, 1H, H1, 4.6), 2.82 (brd, 1H, H-4, 2.7), 2.53 (brt, 1H, H-7, 7.6), 2.44-2.36 (m, 2H, H-3), 2.34 (dd, 1H, H-6, 5.0, 18.7), 2.05 (d, 1H, H-6, 18.7), ${ }^{13} \mathrm{C}-\mathrm{NMR}-125 \mathrm{MHz}\left(\mathrm{CDCl}_{3}+\mathrm{TFA}, \delta \mathrm{ppm}, J \mathrm{~Hz}\right): 216.06$ (C-5), 157.45 $\left(\mathrm{q}, \mathrm{COCF}_{3}, J=42.5\right), 114.39$ (q, $\left.\mathrm{COCF}_{3}, J=282.5\right), 64.96(\mathrm{C}-8), 56.65(\mathrm{C}-2), 52.40(\mathrm{C}-4), 47.03(\mathrm{C}-7)$, 46.84 (C-1), 45.04 (C-6), 33.91 (C-3).

5.NMR spectra of the enantiomer compound $\mathbf{5}$ and of the trifluoroacetylated compound $\mathbf{3 b}$.
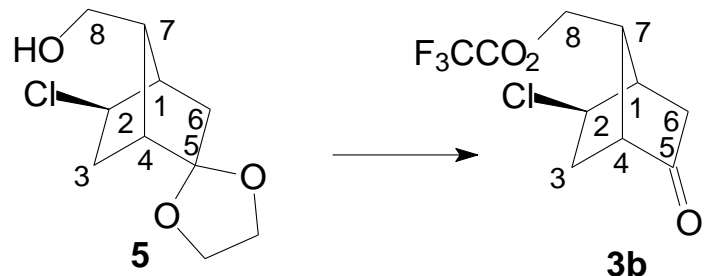

Compound ( \pm )-5a: ${ }^{1} \mathrm{H}-\mathrm{NMR}-500 \mathrm{MHz}\left(\mathrm{CDCl}_{3}, \delta\right.$ ppm, $\left.J \mathrm{~Hz}\right): 3.98(\mathrm{dd}, 1 \mathrm{H}, \mathrm{H}-2,3.7,7.9), 3.93-$ $3.85\left(\mathrm{~m}, 4 \mathrm{H}, \mathrm{OCH}_{2} \mathrm{CH}_{2} \mathrm{O}\right), 3.80(\mathrm{dd}, 1 \mathrm{H}, 6.7,12.0), 3.78(\mathrm{dd}, 1 \mathrm{H}, 6.6,12.0), 2.50(\mathrm{dd}, 1 \mathrm{H}, \mathrm{H}-3,8.0$, 13.9), 2.49 (d, 1H, H-1, 6.6), 2.30 (dd, 1H, H-7, 6.6, 6.7), 2.22 (d, 1H, H-4, 4.1), 1.96 (dt, 1H, H-6, 5.5, 13.9), 1.94 (m, 1H, H-3), 1.92 (s, 1H, H-OH), 1.48 (d, 1H, H-6, 13.9), ${ }^{13} \mathrm{C}-\mathrm{NMR}-125 \mathrm{MHz}\left(\mathrm{CDCl}_{3}, \delta\right.$ ppm, $J$ Hz): $113.84(\mathrm{C}-5), 64.51,64.07\left(\mathrm{OCH}_{2} \mathrm{CH}_{2} \mathrm{O}\right), 60.50(\mathrm{C}-8), 59.92(\mathrm{C}-2), 51.51$ (C-4), 46.40, 46.33 (C-7, C-1), 43.80 (C-6), 33.54 (C-3). 
Compound $( \pm)-5 a$ with TFA for $24 \mathrm{~h}$ gave $3 \mathrm{~b}:{ }^{1} \mathrm{H}-\mathrm{NMR}-500 \mathrm{MHz}\left(\mathrm{CDCl}_{3}+\mathrm{TFA}, \delta \mathrm{ppm}, J \mathrm{~Hz}\right)$ : $4.80(\mathrm{dd}, 1 \mathrm{H}, \mathrm{H}-8,9.3,11.6), 4.71(\mathrm{dd}, 1 \mathrm{H}, \mathrm{H}-8,6.1,11.6), 4.64$ (s, $\left.2 \mathrm{H}, \mathrm{CH}_{2} \mathrm{OH}\right), 4.48$ (brt, $1 \mathrm{H}$, $\left.\mathrm{HOCH}_{2} \mathrm{CH}_{2} \mathrm{O}_{2} \mathrm{CCF}_{3}, 4.5\right), 4.10$ (dd, $\left.1 \mathrm{H}, \mathrm{H}-2,4.6,7.2\right), 4.02$ (t, $\left.1 \mathrm{H}, \mathrm{HOCH}_{2} \mathrm{CH}_{2} \mathrm{O}_{2} \mathrm{CCF}_{3}, 4.5\right), 2.96$ (d, 1H, H-1, 4.6), 2.83 (brd, 1H, H-4, 2.7), 2.54 (brdd, 1H, H-7, 6.7, 8.3), 2.42-2.37 (m, 2H, H-3), 2.36 (dd, $1 \mathrm{H}, \mathrm{H}-6,4.8,18.6), 2.06$ (d, 1H, H-6, 18.6), ${ }^{13} \mathrm{C}-\mathrm{NMR}-125 \mathrm{MHz}\left(\mathrm{CDCl}_{3}+\mathrm{TFA}, \delta \mathrm{ppm}, J \mathrm{~Hz}\right): 216.01$ (C-5), 157.42 (q, $\left.\underline{C O C F}_{3}, J=42.5\right), 114.43\left(\mathrm{q}, \mathrm{COCF}_{3}, J=283.8\right), 114.28$ (q, $\left.\mathrm{COCF}_{3}, J=283.8\right), 68.39$ $\left(\mathrm{HOCH}_{2} \mathrm{CH}_{2} \mathrm{O}_{2} \mathrm{CCF}_{3}\right), 64.98(\mathrm{C}-8), 64.24\left(\mathrm{HOCH}_{2} \mathrm{CH}_{2} \mathrm{OH}\right), 60.12\left(\mathrm{HOCH}_{2} \mathrm{CH}_{2} \mathrm{O}_{2} \mathrm{CCF}_{3}\right), 56.66$ (C-2), 52.44 (C-4), 47.09 (C-7), 46.89 (C-1), 45.10 (C-6), 33.96 (C-3).

6.NMR spectra of the compound $\mathbf{6} \boldsymbol{a}$ and of the trifluoroacetylated compound $\mathbf{6} \boldsymbol{b}$.

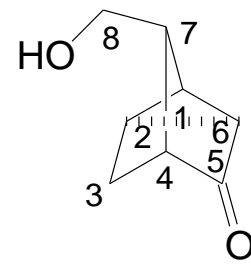

$6 a$

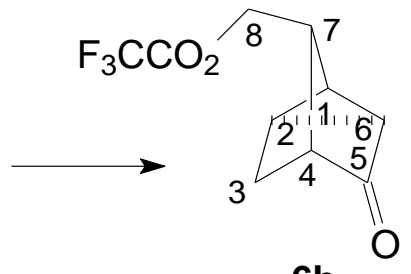

6b

Compound 6a: ${ }^{1} \mathrm{H}-\mathrm{NMR}-500 \mathrm{MHz}\left(\mathrm{CDCl}_{3}, \delta\right.$ ppm, $\left.J \mathrm{~Hz}\right): 3.55(\mathrm{dd}, 1 \mathrm{H}, \mathrm{H}-8,6.6,11.1), 3.50$ (dd, 1H, H-8, 8.2, 11.1), 3.02 (br s, OH), 2.37 (t, 1H, H-7, 7.3), 2.08 (t, 1H, H-2, 4.8), 2.06 (t, 1H, H-1, 5.0), 1.93 (s, $1 \mathrm{H}, \mathrm{H}-4), 1.87$ (s, 2H, H-3), 1.38 (t, 1H, H-6, 5.4), ${ }^{1} \mathrm{H}-\mathrm{NMR}-500 \mathrm{MHz}\left(\mathrm{CDCl}_{3}+\right.$ TFA, $\boldsymbol{t}_{\boldsymbol{0}}, \delta \mathrm{ppm}$, $J \mathrm{~Hz}): 3.73(\mathrm{dd}, 1 \mathrm{H}, \mathrm{H}-8,6.5,11.4), 3.66(\mathrm{dd}, 1 \mathrm{H}, \mathrm{H}-8,7.4,11.4), 2.53(\mathrm{t}, 1 \mathrm{H}, \mathrm{H}-, 7.3), 2.27(\mathrm{t}, 1 \mathrm{H}, \mathrm{H}-$, 4.8), $2.21(\mathrm{t}, 1 \mathrm{H}, \mathrm{H}-, 5.1), 2.09(\mathrm{~s}, 1 \mathrm{H}, \mathrm{H}-), 2.02(\mathrm{~d}, 1 \mathrm{H}, \mathrm{H}-, 11.5), 1.95(\mathrm{~d}, 1 \mathrm{H}, \mathrm{H}-, 11.5), 1.56(\mathrm{t}, 1 \mathrm{H}, \mathrm{H}-$ 5.3), 1.38 (t, 1H, H-, 5.4), ${ }^{13} \mathrm{C}-\mathrm{NMR}-125 \mathrm{MHz}\left(\mathrm{CDCl}_{3}, \delta \mathrm{ppm}, J \mathrm{~Hz}\right): 213.20$ (C-5), 61.37 (C-8), 44.85 (C-7), 39.93 (C-4), 27.46 (C-3), 21.90 (C-6), 20.47 (C-2), 19.87 (C-1).

Compound 6b, formed from $6 \mathrm{a}$ after $48 \mathrm{~h}$ with TFA: ${ }^{1} \mathrm{H}-\mathrm{NMR}-500 \mathrm{MHz}\left(\mathrm{CDCl}_{3}+\mathrm{TFA}, \delta \mathrm{ppm}\right.$, $J \mathrm{~Hz}$ ): 4.32 (dd, 1H, H-8, 6.7, 11.2), 4.28 (dd, 1H, H-8, 8.4, 11.2), 2.66 (dd, 1H, H-7, 7.1, 7.8), 2.25 (t, 1H, H-2, 4.8), 2.19 (t, 1H, H-1, 5.2), 2.03 (d, 1H, H-3, 12.0), 2.02 (s, H, H-4), 1.96 (d, 1H, H-3, 12.0), 1.55 (t, $1 \mathrm{H}, \mathrm{H}-6,5.4)$, From 24 to $120 \mathrm{~h},{ }^{1} \mathrm{H}-\mathrm{NMR}$ spectra remained unchanged, ${ }^{13} \mathrm{C}-\mathrm{NMR}-125 \mathrm{MHz}$ $\left(\mathrm{CDCl}_{3}+\mathrm{TFA}, \delta \mathrm{ppm}, J \mathrm{~Hz}\right): 213.69(\mathrm{C}-5), 157.37\left(\mathrm{q}, \mathrm{COCF}_{3}, J=42.5\right), 114.37\left(\mathrm{q}, \mathrm{COCF}_{3}, J=283.7\right)$, 66.21 (C-8), 40.91 (C-7), 39.92 (C-4), 27.64 (C-3), 22.06 (C-6), 20.47 (C-2), 20.26 (C-1).

7.NMR spectra of the enantiomer compound $7 \boldsymbol{a}$ and of the trifluoroacetylated compound $7 \boldsymbol{b}$.

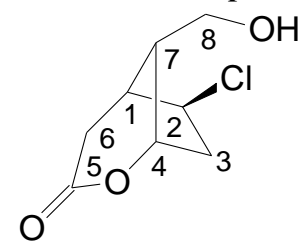

$7 a$

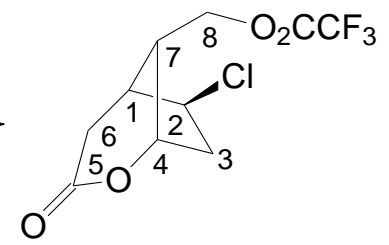

$7 b$

Compound 7a: ${ }^{1} \mathrm{H}-\mathrm{NMR}-500 \mathrm{MHz}\left(\mathrm{CDCl}_{3}, \delta\right.$ ppm, $J \mathrm{~Hz}$ ): 4.92 (brs, $\left.1 \mathrm{H}, \mathrm{H}-4\right), 4.27$ (dd, $1 \mathrm{H}, \mathrm{H}-2$, 4.2, 8.1), 3.86 (dd, 1H, H-8, 8.8, 11.5), 3.84 (dd, 1H, H-8, 7.0, 11.5), 2.98 (dd, 1H, H-3, 8.1, 16.6), 2.85 (dd, 1H, H-6, 5.7, 18.9), 2.70 (m, 1H, H-1), 2.69 (d, 1H, H-6, 18.9), 2.49 (t, 1H, H-7, 8.0), 2.43 (dt, 1H, $\mathrm{H}-3,4.4,16.6),{ }^{13} \mathrm{C}-\mathrm{NMR}-125 \mathrm{MHz}\left(\mathrm{CDCl}_{3}, \delta \mathrm{ppm}, J \mathrm{~Hz}\right): 168.62$ (C-5), 82.08 (C-4), 60.95 (C-8), 59.43 (C-2), 50.47 (C-7), 44.39 (C-1), 44.24 (C-3), 40.56 (C-6). (For esters of 7a see reference [17]).

The compound $7 \mathrm{~b}$, after $24 \mathrm{~h}$ with TFA of the compound $7 \mathrm{~b}:{ }^{1} \mathrm{H}-\mathrm{NMR}-500 \mathrm{MHz}\left(\mathrm{CDCl}_{3}+\mathrm{TFA}\right.$, $\delta$ ppm, $J$ Hz): 5.03 (brdd, 1H, H-4, 1.9, 4.1), 4.65 (dd, 1H, H-8, 6.6, 11.8), 4.61 (dd, 1H, H-8, 10.0, 11.8), 4.37 (dd, 1H, H-2, 4.0, 8.1), 3.13 (dd, 1H, H-3, 8.0, 17.2), 3.04 (dd, 1H, H-6, 5.6, 19.4), 2.86 (d, 1H, H6, 19.4), 2.83 (m. 1H, H-1), 2.78 (t, 1H, H-7, 8.0), 2.57 (dt, 1H, H-3, 4.3, 17.2), ${ }^{13} \mathrm{C}-\mathrm{NMR}-125 \mathrm{MHz}$ $\left(\mathrm{CDCl}_{3}+\mathrm{TFA}, \delta \mathrm{ppm}, J \mathrm{~Hz}\right): 173.68(\mathrm{C}-5), 157.53\left(\mathrm{q}, \mathrm{COCF}_{3}, J=43.7\right), 114.24\left(\mathrm{q}, \mathrm{COCF}_{3}, J=282.5\right)$, 83.07 (C-4), 65.32 (C-8), 58.13 (C-2), 46.58 (C-7), 44.40 (C-1), 43.82 (C-3), 39.75 (C-6). 
8.NMR spectra of the compound $8 \boldsymbol{a}$ and of the trifluoroacetylated compound $8 \boldsymbol{8}$.
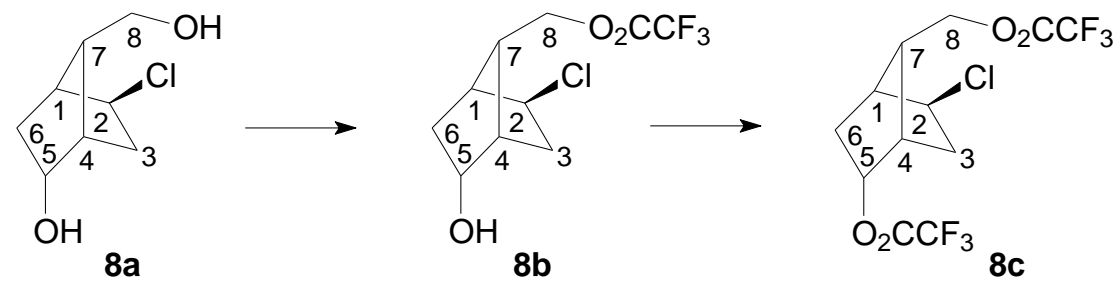

Compound 8a: ${ }^{1} \mathrm{H}-\mathrm{NMR}-500 \mathrm{MHz}\left(\mathrm{CDCl}_{3}, \delta\right.$ ppm, $\left.J \mathrm{~Hz}\right): 4.23(\mathrm{dt}, 1 \mathrm{H}, \mathrm{H}-5,3.5,9.9), 4.03(\mathrm{dd}, 1 \mathrm{H}$, H-2, 3.6, 8.0), 3.96 (dd, 1H, H-8, 7.8, 11.4), 3.91 (dd, 1H, H-8, 6.5, 11.4), 2.79 (dd, 1H, H-3, 8.0, 14.6), 2.46 (d, 1H, H-1, 5.0), 2.39 (t, 1H, H-4, 4.1), 2.14 (ddd, 1H, H-6, 5.2, 9.9, 13.7), 2.00 (t, 1H, H-7, 7.9), 1.96 (brt, 1H, H-3, 3.6, 14.6), 1.59 (s, 2H, OH), 0.91 (dd, 1H, H-6, 3.0, 13.7), ${ }^{13} \mathrm{C}-\mathrm{NMR}-125 \mathrm{MHz}$ $\left(\mathrm{CDCl}_{3}, \delta \mathrm{ppm}, J \mathrm{~Hz}\right): 70.25$ (C-5), 60.78 (C-2), 60.69 (C-8), 51.38 (C-7), 47.68 (C-1), 44.88 (C-4), 39.50 (C-6), 32.48 (C-3). (See NMR spectra in DMSO-d6 in ref. [15,18]).

Compound 8c, formed from 8 a after $24 \mathrm{~h}$ with TFA: ${ }^{1} \mathrm{H}-\mathrm{NMR}-500 \mathrm{MHz}\left(\mathrm{CDCl}_{3}+\mathrm{TFA}, \delta \mathrm{ppm}\right.$, $J$ Hz): 5.20 (dt, 1H, H-5, 3.5, 10.0), 4.81 (dd, 1H, H-8, 9.3, 11.5), 4.70 (dd, 1H, H-8, 6.4, 11.5), 4.05 (dd, 1H, H-2, 3.9, 8.0), 2.83 (t, 1H, H-4, 4.1), 2.67 (dd, 1H, H-3, 8.1, 15.3), 2.64 (d, 1H, H-1, 4.8), 2.37 (ddd, 1H, H-6, 5.0, 10.1, 14.6), 2.25 (brt, 1H, H-7, 7.8), 2.13 (brdt, 1H, H-3, 3.8, 15.3), 1.26 (dd, 1H, H-6, 3.0, 14.6), ${ }^{13} \mathrm{C}-\mathrm{NMR}-125 \mathrm{MHz}\left(\mathrm{CDCl}_{3}+\mathrm{TFA}, \delta \mathrm{ppm}, J \mathrm{~Hz}\right): 158.24$ (q, $\mathrm{COCF}_{3}$ in $\left.\mathrm{C}_{5}, J=43.7\right), 157.80$ (q, $\mathrm{COCF}_{3}$, in $\left.\mathrm{C}_{8}, J=42.5\right), 114.54$ (q, $\mathrm{COCF}_{3}$, in $\left.\mathrm{C}_{5}, J=282.5\right), 114.41$ (q, $\mathrm{COCF}_{3}$, in $\left.\mathrm{C}_{8}, J=283.7\right), 76.79$ (C-5), 65.89 (C-8), 58.39 (C-2), 47.63 (C-1), 47.63 (C-7), 42.95 (C-4), 36.31 (C-6), 32.83 (C-3).

9.NMR spectra of the ent-Corey compound $9 \boldsymbol{a}$ and of the bis-trifluoroacetylated compound $9 \mathrm{c}$.
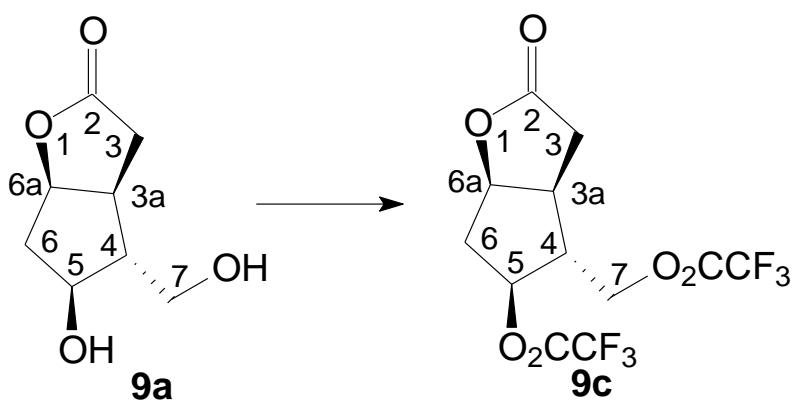

Compound 9a: ${ }^{1} \mathrm{H}-\mathrm{RMN}-500 \mathrm{MHz}\left(\mathrm{CDCl}_{3}, \delta\right.$ ppm, $\left.J \mathrm{~Hz}\right)$ : 4.93 (dt, 1H, H-6a, 2.7, 6.8), 4.19 (q, 1H, H5, 6.3), 3.78 (dd, 1H, H-7, 5.3, 10.4), 3.66 (dd, 1H, H-7, 7.3, 10.4), 2.81 (dd, 1H, H-3, 9.9, 18.1), 2.62 (m, 1H, H-3a), 2.53 (dd, 1H, H-3, 2.0, 18.1), 2.46 (dt, 1H, H-6, 6.6, 14.9), 2.05-2.00 (m, 2H, H-4, H-6), ${ }^{13} \mathrm{C}-\mathrm{RMN}-125 \mathrm{MHz}\left(\mathrm{CDCl}_{3}, \delta \mathrm{ppm}\right): 180.63$ (COO), 83.29 (C-6a), 75.65 (C-5), 63.73 (C-7), 55.02 (C4), 40.81 (C-6), 39.44 (C-3a), 35.18 (C-3).

Compound 9c, formed from 9a after $72 \mathrm{~h}$ with TFA: ${ }^{1} \mathrm{H}-\mathrm{RMN}-500 \mathrm{MHz}\left(\mathrm{CDCl}_{3}+\mathrm{TFA}, 72 \mathrm{~h}, \delta \mathrm{ppm}\right.$, $J$ Hz): 5.33 (dt, 1H, H-6a, 3.3, 5.9), 5.27 (brdt, 1H, H-5, 1.3, 6.7), 4.45 (dd, 1H, H-7, 5.4, 11.5), 4.39 (dd, $1 \mathrm{H}, \mathrm{H}-7,6.2,11.5), 3.14$ (dd, 1H, H-3, 10.2, 18.9), 2.96 (m, 1H, H-3a), 2.78 (dd, 1H, H-3, 2.0, 18.9), 2.66 (dt, 1H, H-4, 5.4, 10.0), 2.59 (dt, 1H, H-6, 6.1, 16.4), 2.47 (brdd, 1H, H-6, 3.1, 16.4), ${ }^{13} \mathrm{C}-\mathrm{RMN}-$ $125 \mathrm{MHz}\left(\mathrm{CDCl}_{3}+\mathrm{TFA}, 72 \mathrm{~h}, \delta \mathrm{ppm}\right): 176.76(\mathrm{COO}), 157.41$ (q, $\left.\mathrm{COCF}_{3}, J=49.5\right), 114.26\left(\mathrm{q}, \mathrm{COCF}_{3}\right.$, $J=282.8), 157.10\left(\mathrm{q}, \underline{\mathrm{COCF}}_{3}, J=42.4\right), 114.61\left(\mathrm{q}, \mathrm{COCF}_{3}, J=283.4\right), 85.83(\mathrm{C}-6 \mathrm{a}), 80.60(\mathrm{C}-5), 66.57$ (C-7), 50.61 (C-4), 40.39 (C-3a), 37.59 (C-6), 35.93 (C-3).

10.NMR spectra of the ent-Corey compound $10 \mathrm{a}$ and of the bis-trifluoroacetylated compound $10 \mathrm{~b}$.

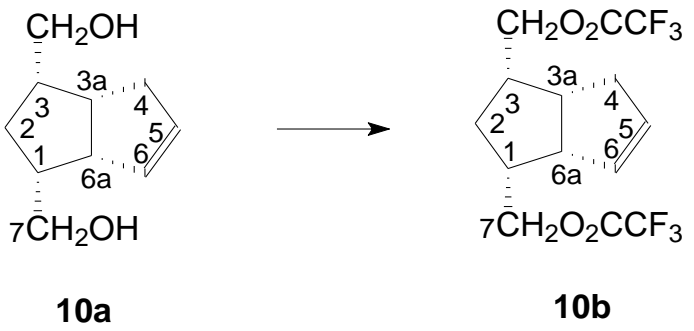


Compound 10a: ${ }^{1} \mathrm{H}-\mathrm{RMN}-500 \mathrm{MHz}\left(\mathrm{CDCl}_{3}, \delta \mathrm{ppm}, J \mathrm{~Hz}\right): 5.73(\mathrm{~m}, 1 \mathrm{H}, \mathrm{H}-6), 5.59$ (dd, $1 \mathrm{H}, \mathrm{H}-5$, 2.4, 5.4), 3.67 (d, 2H, H-8, 7.4), 3.62 (dd, 1H, H-7, 7.6, 10.4), 3.57 (dd, 1H, H-7, 6.8, 10.4), 3.30 (brt, 1H, H-6a, 7.6), 2.90 (m, 1H, H-3a), 2.32 (dd, 1H, H-4, 9.7, 17.0), 2.25-2.17 (m, 3H, H-1, H-3, H-3a), 2.01 (brs, 2H, HO), 1.72 (dt, 1H, H-2, 5.4, 12.0), 0.85 (q, 1H, H-2, 12.0), ${ }^{13} \mathrm{C}-\mathrm{RMN}-125 \mathrm{MHz}\left(\mathrm{CDCl}_{3}\right.$, $\delta$ ppm): 132.32 (C-6), 129.73 (C-5), 64.38, 64.17 (C-7, C-8), 51.87 (C-6a), 45.96, 45.33 (C-1, C-3), 41.93 (C-3a), 33.03 (C-4), 30.80 (C-2). (NMR spectra in DMSO, ref. [19]).

Compound 10b: ${ }^{1} \mathrm{H}-\mathrm{RMN}-500 \mathrm{MHz}\left(\mathrm{CDCl}_{3}+\mathrm{TFA}, 24 \mathrm{~h}, \delta\right.$ ppm, $\left.J \mathrm{~Hz}\right): 5.82$ (brd, $\left.1 \mathrm{H}, \mathrm{H}-6,3.5\right)$, 5.51 (brd, 1H, H-5, 3.7), 4.42 (2, 2H, H-8, 7.6), 4.33 (2, 2H, H-7, 7.6), 3.38 (brt, 1H, H-6a, 8.1), 2.99 (m, 1H, H-3a), 2.50-2.43 (m, 2H, H-1, H-3), 2.39 (dd, 1H, H-4, 9.7, 17.4), 2.19 (brd, 1H, H-4, 17.4), 1.82 (dt, $1 \mathrm{H}, \mathrm{H}-2,5.4,12.3), 1.03$ (q, $1 \mathrm{H}, \mathrm{H}-2,12.3),{ }^{13} \mathrm{C}-\mathrm{RMN}-125 \mathrm{MHz}\left(\mathrm{CDCl}_{3}+\mathrm{TFA}, 24 \mathrm{~h}, \delta \mathrm{ppm}\right)$ : 158.06 (q, $\left.\mathrm{COCF}_{3}, J=42.1\right), 158.04$ (q, $\left.\mathrm{COCF}_{3}, J=42.1\right), 133.51(\mathrm{C}-6), 128.30$ (C-5), $114.52(\mathrm{q}$, $\left.\mathrm{COCF}_{3}, J=282.5\right), 69.67,69.37$ (C-7, C-8), 51.61 (C-6a), 42.17 (C-3a), 41.71, 41.14 (C-1, C-3), 33.29 (C-4), $30.68(\mathrm{C}-2)$.

11.NMR spectra of the optically active enone $13 \boldsymbol{a}$ and of the trifluoroacetylated compound $\mathbf{1 3 b}$.
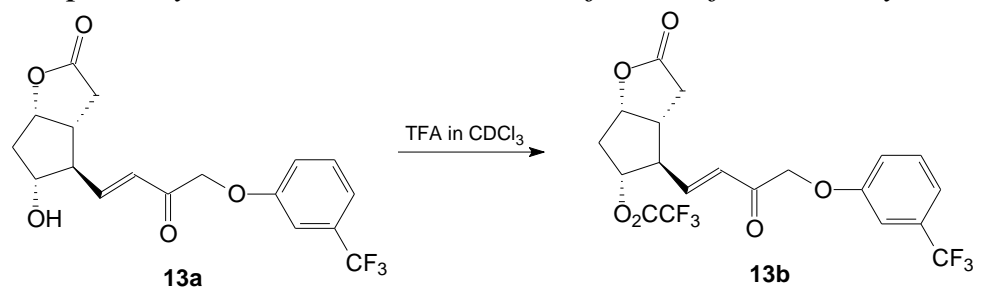

Compound 13a: ${ }^{1} \mathrm{H}-\mathrm{NMR}-300 \mathrm{MHz}\left(\mathrm{CDCl}_{3}, \delta \mathrm{ppm}, J \mathrm{~Hz}\right): 7.22(\mathrm{t}, 1 \mathrm{H}, \mathrm{H}-22,8.2), 7.00(\mathrm{dd}, 1 \mathrm{H}, \mathrm{H}-$ 21, 1.0, 8.2), 6,90 (d, 1H, H-19, 2.2), 6.85 (dd, 1H, H-13, 8.4, 15.7), 6.79 (dd, 1H, H-23, 2.4, 8.2), 6.54 (d, 1H, H-14, 15.7), 4.97 (dt, 1H, H-9, 2.7, 6.8), 4.68 (s, 2H, H-16), 4.16 (q, 1H, H-11, 6.6), 3.04 (dd, 1H, H-7, 9.9, 17.6), 2.95 (m, 1H, H-8), 2.69 (d + TFA, 1H, H-7, 17.6), 2.58 ( dt + TFA, 1H, H-10, 6.8, 15.4), 2.60 (q, $1 \mathrm{H}, \mathrm{H}-12,7.3), 2.08$ (ddd, $1 \mathrm{H}, \mathrm{H}-10,2.7,6.6,15.4),{ }^{13} \mathrm{C}-\mathrm{NMR}-75 \mathrm{MHz}\left(\mathrm{CDCl}_{3}, \delta \mathrm{ppm}\right)$ : 194.59 (C-15), 176.28 (C-6), 158.51 (C-18), 146.68 (C-14), 135.11 (C-20), 130.50 (C-22), 126.51 (C13), 122.11 (C-21), 115.15 (C-19), 113.02 (C-23), 82.59 (C-9), 76.55 (C-11), 72.21 (C-16), 56.35 (C12), 42.39 (C-8), 40.39 (C-10), 34.44 (C-7).

\section{Compound 13a with TFA, after 5 days:}

${ }^{1} \mathrm{H}-\mathrm{NMR}-300 \mathrm{MHz}\left(\mathrm{CDCl}_{3}, \delta \mathrm{ppm}, J \mathrm{~Hz}\right): 7.24$ (t, 1H, H-22, 8.3), 7.04 (brd, 1H, H-21, 8.3), 6.99 (dd, 1H, H-13, 8.1, 15.8), 6,90 (dd, 1H, H-19, 2.1, 2.4), 6.78 (dd, 1H, H-23, 2.4, 8.3), 6.64 (d, 1H, H-14, 15.8), 5.34-5.27 (m, 2H, H-9, H-11), 4.84 (s, 2H, H-16), 3.12-3.02 (m, 3H, H-7, H-8, H-12), 2.72 (1H, $\mathrm{H}-7,17.0), 2.72$ ( dt, 1H, H-10, 6.4, 16.1), 2.41 (dd, 1H, H-10, 4.4, 16.1), ${ }^{13} \mathrm{C}-\mathrm{NMR}-75 \mathrm{MHz}\left(\mathrm{CDCl}_{3}, \delta\right.$ ppm): $198.69(\mathrm{C}-15), 181.17 .28(\mathrm{C}-6), 162.78\left(\mathrm{q}, \mathrm{COCF}_{3}, J=43.5\right), 157.63(\mathrm{C}-18), 146.61(\mathrm{C}-14)$, 135.11 (C-20), 130.79 (C-22), 126.86 (C-13), 122.95 (C-21), 115.19 (C-19), 114.34 (q, $\mathrm{COCF}_{3}, J=$ 282.2), 112.98 (C-23), 85.53 (C-9), 81.73 (C-11), 71.19 (C-16), 54.01 (C-12), 42.79 (C-8), 37.11 (C10), 35.31 (C-7).

12.NMR spectra of the optically active diol compound $\mathbf{1 4 a}$ and of the bis-trifluoroacetylated compound $14 c$.
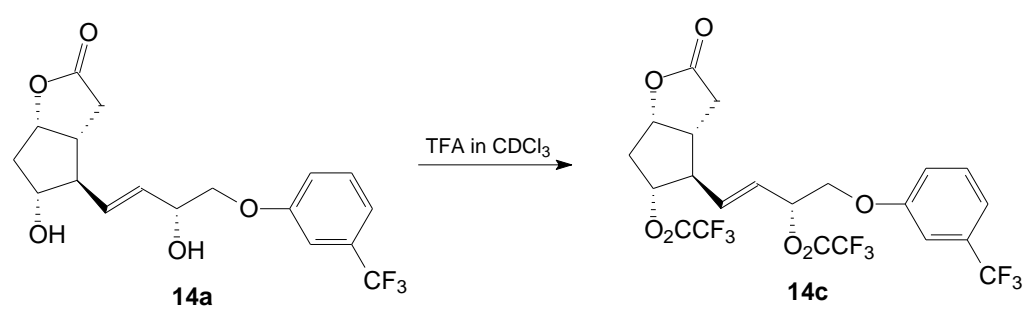

Compound 14a: ${ }^{1} \mathrm{H}-\mathrm{NMR}-300 \mathrm{MHz}\left(\mathrm{CDCl}_{3}, \delta \mathrm{ppm}, J \mathrm{~Hz}\right): 7.40(\mathrm{t}, 1 \mathrm{H}, \mathrm{H}-22,8.0), 7.24(\mathrm{~d}, 1 \mathrm{H}, \mathrm{H}-$ 21, 8.0), 7.14 (brs, 1H, H-19), 7.09 (brd, 1H, H-23, 8.0), 5.77-5.67 (m, 2H, H-13, H-14), 4.92 (td, 1H, H-9, 6.9, 3.1), 4.54 (dt, 1H, H-15, 3.5, 7.1), 4.03 (dd, 1H, H-16, 3.3, 9.4), 4.00 (dd, 1H, H-11, 7.1, 9.4), 3.93 (dd, 1H, H-16, 7.6, 9.4), 2.75 (dd, 1H, H-7, 9.5, 17.7), 2.63 (brq, 1H, H-8, 8.4), 2.54 (dd, 1H, H-10, 7.1, 14.6), 2.45 (d, 1H, H-7, 17.7), 2.35 (m, 1H, H-12), 1.98 (ddd, 1H, H-10, 2.8, 7.4, 14.6), ${ }^{13} \mathrm{C}-$ 
NMR-75MHz (CDCl 3 , $\delta$ ppm): 176.68 (C-6), 158.42 (C-18), 132.64 (C-14 or 13), 130.98 C-13 or 14), 130.16 (C-22), 118.06 (C-19), 111.37 (C-23), 82.42 (C-9), 76.52 (C-11), 71.81 (C-16), 70.50 (C-15), 56.35 (C-12), 42.49 (C-8), 39.85 (C-10), 34.22 (C-7).

Compound 14a with TFA, after 4 days: ${ }^{1} \mathrm{H}-\mathrm{NMR}-300 \mathrm{MHz}\left(\mathrm{CDCl}_{3}+\mathrm{TFA} 3\right.$ days, $\left.\delta \mathrm{ppm}, J \mathrm{~Hz}\right)$ : 7.42 (t, 1H, H-22, 8.0), 7.28 (d, 1H, H-21, 8.0), 7.11 (brt, 1H, H-19, 2.1), 7.07 (dd, 1H, H-23, 2.1, 8.0), 5.87-5.73 (m, 3H, H-13, H-14, H-15), 5.28-5.22 (m, 2H, H-11, H-9), 4.21 (d, 2H, H-16, 4.7), 3.07 (dd, 1H, H-7, 9.8, 18.5), 2.95 (m, 1H, H-8, 9.8), 2.85 (q, 1H, H-12, 6.3), 2.68 (d, 1H, H-7, 18.6), 2.66 (dt, $1 \mathrm{H}, \mathrm{H}-10,6.5,16.0), 2.34$ (dd, $1 \mathrm{H}, \mathrm{H}-10,4.2,16.0),{ }^{13} \mathrm{C}-\mathrm{NMR}-75 \mathrm{MHz}\left(\mathrm{CDCl}_{3}, \delta \mathrm{ppm}\right): 181.62(\mathrm{C}-6)$, 157.97 (C-18), 134.58 (C-14), 130.42 C-13), 126.71 (C-22), 118.92 (C-21), 118.36 (C-19), 111.45 (C23), 85.42 (C-9), 81.74 (C-11), 76.39 (C-15), 68.47 (C-16), 53.79 (C-12), 42.56 (C-8), 36.66 (C-10), 35.16 (C-7). In this experiment, the amount of $\mathbf{1 4 a}$ was small and the time acquisition of the ${ }^{13} \mathrm{C}-\mathrm{NMR}$ spectra was short to put in evidence the carbon atoms $\mathrm{C}_{20}, \mathrm{C}_{21}$ and $\mathrm{CF}_{3}$ for the compound $\mathbf{1 4 a}$, and the carbon atoms $\mathrm{C}_{20}, \mathrm{CF}_{3}$ and the carbon atoms of the trifluoroacetyl groups for the compound 14c.

13.NMR spectra of the optically active diol compound 15a and of the bis-trifluoroacetylated compound 15c.
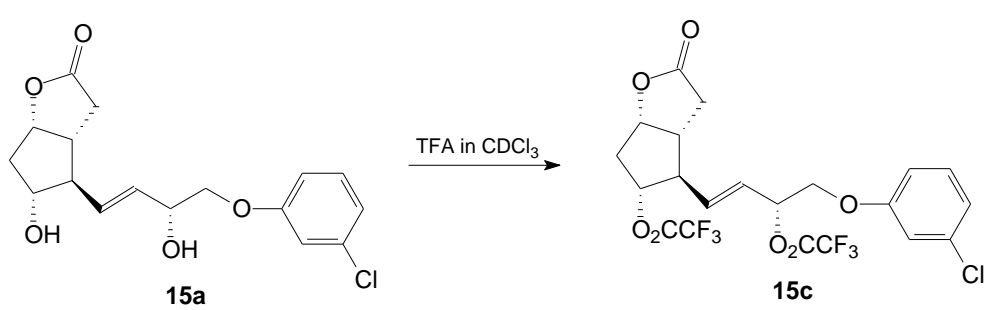

Compound 15a: ${ }^{1} \mathrm{H}-\mathrm{NMR}-300 \mathrm{MHz}\left(\mathrm{CDCl}_{3}, \delta \mathrm{ppm}, J \mathrm{~Hz}\right): 7.20(\mathrm{t}, 1 \mathrm{H}, \mathrm{H}-22,8.2), 6.96$ (dd, $1 \mathrm{H}$, H-21, 2.4, 8.2), 6.91 (t, 1H, H-19, 2.3), 6.79 (dd, 1H, H-23, 2.4, 8.2), 5.71 (dd, 1H, H-13, 4.8, 16.5), 5.67 (dd, 1H, H-14, 4.8, 16.5), 4.91 (td, 1H, H-9, 7.0, 3.1), 4.50 (dt, 1H, H-15, 3.6, 7.4), 4.00 (t, 1H, H-11, 7.6), 3.99 (dd, 1H, H-16, 3.8, 9.5), 3.87 (dd, 1H, H-16, 7.7, 9.5), 2.74 (dd, 1H, H-7, 9.5, 18.9), 2.62 (brq, 1H, H-8, 9.0), 2.53 (dd, 1H, H-10, 7.0, 14.7), 2.43 (dd, 1H, H-7, 1.2, 18.9), 2.34 (m, 1H, H-12), 1.96 (ddd, $1 \mathrm{H}, \mathrm{H}-10,3.1,7.6,14.6),{ }^{13} \mathrm{C}-\mathrm{NMR}-75 \mathrm{MHz}\left(\mathrm{CDCl}_{3}, \delta \mathrm{ppm}\right): 176.74(\mathrm{C}-6), 159.02$ (C-18), 134.97 (C-20), 132.69 (C-14 or 13), 130.06 C-13 or 14), 130.38 (C-22), 121.57 (C-21), 115.06 (C-19), 113.06 (C-23), 82.41 (C-9), 76.42 (C-11), 71.74 (C-16), 70.50 (C-15), 56.32 (C-12), 42.43 (C-8), 39.77 (C-10), 34.18 (C-7).

Compound 15a with TFA, after 4 days: ${ }^{1} \mathrm{H}-\mathrm{NMR}-300 \mathrm{MHz}\left(\mathrm{CDCl}_{3}+\mathrm{TFA} 4\right.$ days, $\left.\delta \mathrm{ppm}, J \mathrm{~Hz}\right)$ : 7.23 (t, 1H, H-22, 8.2), 7.01 (brd, 1H, H-21, 7.4), 6.89 (t, 1H, H-19, 2.1), 6.78 (dd, 1H, H-23, 2.3, 8.2), 5.91-5.72 (m, 3H, H-13, H-14, H-15), 5.27-5.22 (m, 2H, H-19, H-11), 4.17 (d, 2H, H-16, 4.7), 3.07 (dd, $1 \mathrm{H}, \mathrm{H}-7,9.9,18.6), 2.95$ (m, 1H, H-8), 2.84 (q, 1H, H-12, 6.3), 2.68 (d, 1H, H-7, 18.6), 2.64 (dt, 1H, H10, 6.6, 16.0), 2.34 (brdd, 1H, H-10, 4.2, 16.0), ${ }^{13} \mathrm{C}-\mathrm{NMR}-75 \mathrm{MHz}\left(\mathrm{CDCl}_{3}, \delta \mathrm{ppm}\right): 181.61$ (C-6), 161.58 $\left.\left(\mathrm{q}, \mathrm{CF}_{3} \mathrm{CO}, J_{\mathrm{CF}}=43.5\right), 158.46(\mathrm{C}-18), 135.28(\mathrm{C}-20), 134.55(\mathrm{C}-14), 130.62 \mathrm{C}-13\right), 126.70(\mathrm{C}-22)$, $122.44(\mathrm{C}-21), 115.31(\mathrm{C}-19), 114.34\left(\mathrm{q}, \mathrm{CF}_{3} \mathrm{CO}, J_{\mathrm{CF}}=282.8\right), 113.23(\mathrm{C}-23), 85.44(\mathrm{C}-9), 81.79(\mathrm{C}-$ 11), 76.37 (C-15), 68.43 (C-16), 53.79 (C-12), 42.53 (C-8), 36.67 (C-10), 35.20 (C-7).

14.NMR spectra of the compound $12 \mathrm{a}$ and of the bis-trifluoroacetylated compound $12 \mathrm{c}$.

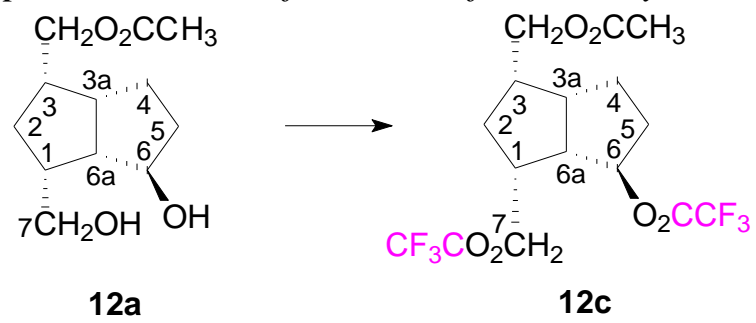

Compound 12a: ${ }^{1} \mathrm{H}-\mathrm{NMR}$ (DMSO-d6, $\delta$ ppm, $J$ Hz): 3.97 (d, 2H, H-8, 7.6), 3.77 (q, 1H, H-6, 7.1) ( $\mathrm{t}$ +TFA, 10.8), 3.62 (dd with THF, 1H, H-7, 6.7, 10.8), 2.57 (dt + TFA, 1H, H-3a, 6.2, 9.0), 2.20 (dt, $1 \mathrm{H}, 6 \mathrm{a}, 6.3,8.8), 2.08-1.99$ (m, 2H, H-1, H-3), 1.98 (s, 3H, CH $\mathrm{CH}_{3}, 1.67$ (m, 1H, H-5), 1.61 (dt, 1H, H-2, 5.4, 12.4), 1.49 (m, 1H, H-4), 1.28 (m, 1H, H-5), 1.14 (m, 1H, H-4), 0.86 (q, 1H, H-2, 12.4), ${ }^{13} \mathrm{C}-\mathrm{NMR}$ 
(DMSO-d6, $\delta$ ppm): 170.37 (COO), 72.69 (C-6), 64.66 (C-7), 61.90 (C-8), 51.80 (C-6a), 43.82 (C-3), 42.48 (C-1), 40.46 (C-3a) 35.39 (C-5), 31.01 (C-2), 23.03 (C-4), $20.72\left(\mathrm{CH}_{3}\right)(\mathrm{NMR}$ spectra in DMSO, [20]).

NMR spectra of the compound 12a + TFA, after 7 days, are presented below:

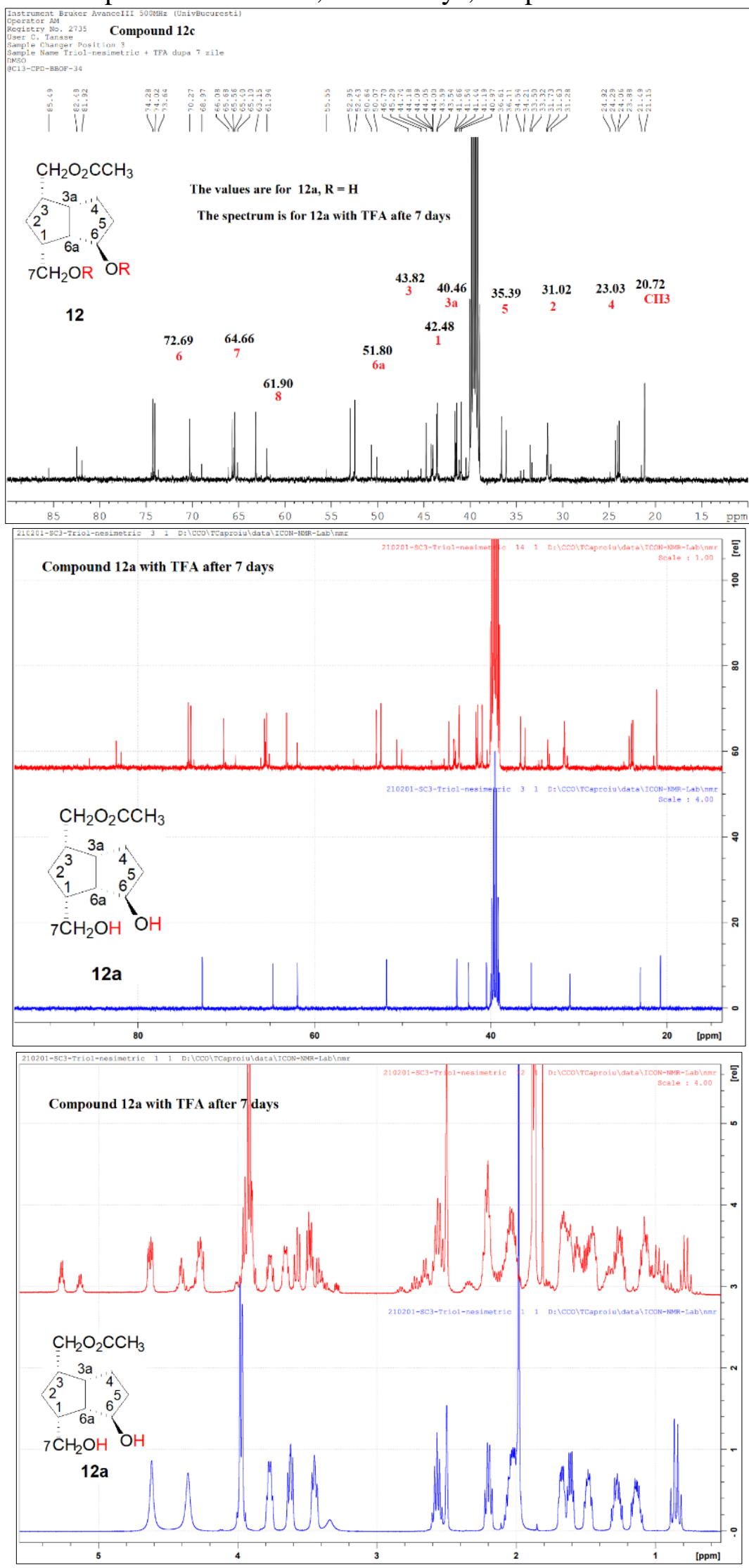


The un-symmetric triol 12 was esterified with trifluoroacetic anhydride in TFA (in NMR tube) and the complete esterification proceeded in $4 \mathrm{~h}$. The signals of the bis-triflouroacetylated compound 12c are described below: ${ }^{1} \mathrm{H}-\mathrm{NMR}\left(\mathrm{TFA}+\left(\mathrm{CF}_{3} \mathrm{CO}\right)_{2} \mathrm{O}, \delta \mathrm{ppm}, J \mathrm{~Hz}\right): 5.18$ (dt, 1H, H-6, 5.5, 6.6), 4.54 (dd, 1H, H-7, 6.6, 11.3), 4.45 (dd, 1H, H-7, 8.0, 11.3), 4.26-4.20 (m, 2H, H-8), 2.92 (qv, 1H, H-3a, 8.2), 2.83 (td, 1H, H-6a, 14.7, 9.1), 2.48 (hp, 1H, H-1, 6.1), 2.36 (m, 1H, H-3), 2.18 (s, 3H, CH $), 2.13$ (m, 1H, H5), 1.91-1.68 (m, 3H, H-2, H-4, H-5), 1.42 (m, 1H, H-4), 1.11 (q, 1H, H-2, 12.6), ${ }^{13} \mathrm{C}-\mathrm{NMR}$ (TFA + $\left.\left(\mathrm{CF}_{3} \mathrm{CO}\right)_{2} \mathrm{O}, \delta \mathrm{ppm}\right): 176.05(\mathrm{COO}), 157.96\left(J_{\mathrm{C}-\mathrm{F}}=42.8\right), 157.87\left(\mathrm{COCF}_{3}, J_{\mathrm{C}-\mathrm{F}}=42.8\right), 114.44\left(\mathrm{COCF}_{3}\right.$, $\left.J_{\mathrm{C}-\mathrm{F}}=282.0\right), 80.72(\mathrm{C}-6), 67.70(\mathrm{C}-7), 66.49(\mathrm{C}-8), 49.45(\mathrm{C}-6 \mathrm{a}), 43.16(\mathrm{C}-3 \mathrm{a}), 40.31(\mathrm{C}-1), 39.92(\mathrm{C}-$ 3), $32.65(\mathrm{C}-5), 30.81(\mathrm{C}-2), 23.74(\mathrm{C}-4), 20.82\left(\mathrm{CH}_{3}\right)$ (NMR spectra in DMSO, [20]).

15.NMR spectra of the ent-Corey compound 11 a and of the bis-trifluoroacetylated compound 11c.

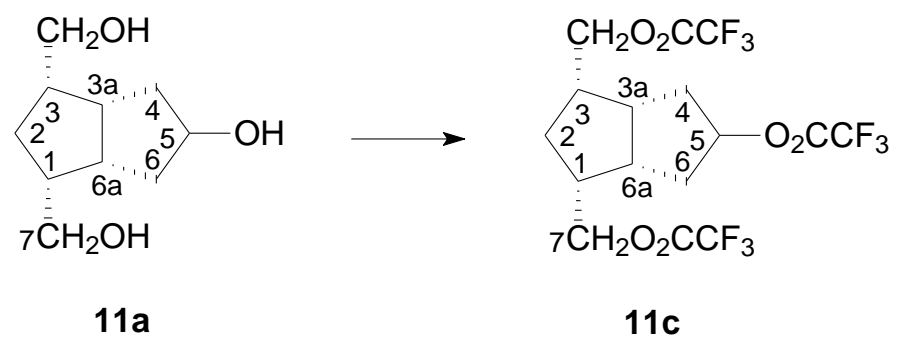

Compound 11a: ${ }^{1} \mathrm{H}-\mathrm{NMR}$ (DMSO-d6, $\delta \mathrm{ppm}, J \mathrm{~Hz}$ ): 4.26 (t, 2H, HO-C $7,8,5.0$, deuterable), 4.16 (d, 1H, HO-C 5 , 2.8, deuterable), 4.09 (bs, 1H, H-5), 3.36 (d, 2H, H-8, 11.6), 3.33 (brd, 2H, H-7, 7.3), 2.63 (hext, 2H, H-6a, H-3a, 8.5), 1.95 (hept, 2H, H-1, H-3, 7.2), 1.55 (dt, 1H, H-2, 5.3, 12.4), 1.51-1.47 (m, 2H, H-4, H-6), 1.27-1.20 (m, 2H, H-4, H-6), 0.71 (q, 1H, H-2, 12.4), ${ }^{1} \mathrm{H}-\mathrm{NMR}$ (DMSO-d6 + TFA, $\mathrm{t}_{0}, \delta$ ppm, $J$ Hz): 4.10 (brs, 1H, H-5), 3.34 (d, 4H, 2H-7, 2H-8, 7.3, 11.0), 2.64 (hex, 2H, H-6a, H-3a, 8.5), 1.95 (hept, 2H, H-1, H-3, ), 1.55 (dt, 1H, H-2, 5.2, 12.4), 1.51-1.47 (m, 2H, H-4, H-6), 1.28-1.20 (m, 2H, H-4, H-6), 0.71 (q, 1H, H-2, 12.4), ${ }^{13} \mathrm{C}-\mathrm{NMR}$ (DMSO-d6, $\delta$ ppm): 72.90, 72.78 (C-5), 61.95, 61.83 (C-7, C-8), 44.32, 44.27 (C-1, C-3), 41.98 (C-3a, C-6a), 35.30, 35.25 (C-4, C-6), 30.63 (C-2). (NMR spectra in DMSO, [20]).

The compound 11a was not completely esterified, even after 10 days and with a significant excess of TFA. No proton and carbon NMR spectra couldn't be decipherable, as in the case of 10a + TFA.

The symmetric triol 11 was esterified with trifluoacetic anhydride in TFA (in NMR tube) and the complete esterification proceeded in $4 \mathrm{~h}$. The signals of the tri-esterified compound 11c are described below: ${ }^{1} \mathrm{H}-\mathrm{NMR}\left(\mathrm{TFA}+\left(\mathrm{CF}_{3} \mathrm{CO}\right)_{2} \mathrm{O}, \delta \mathrm{ppm}, J \mathrm{~Hz}\right): 5.53(\mathrm{t}, 1 \mathrm{H}, \mathrm{H}-5,3.7), 4.43$ (dd, 2H, H-7, H-8, 7.3, 11.1), 4.35 (dd, 2H, H-7, H-8, 3.0, 11.1), 3.04-2.91 (m, 2H, H-3a, H-6a), 2.58-2.44 (m, 2H, H-1, H-3), 2.05 (brd, 1H, H-4 or H-h, 4.2), 2.02 (brd, 1H, H-6 or H-4, 7.1), 1.89 (dt, 1H, H-2, 11.5, 5.3), 1.61-1.55 $(\mathrm{m}, 2 \mathrm{H}, \mathrm{H}-4, \mathrm{H}-6), 1.16$ (q, $1 \mathrm{H}, \mathrm{H}-2,11.5),{ }^{13} \mathrm{C}-\mathrm{NMR}\left(\mathrm{TFA}+\left(\mathrm{CF}_{3} \mathrm{CO}\right)_{2} \mathrm{O}, \delta \mathrm{ppm}\right): 158.13\left(\mathrm{COCF}_{3}\right.$ in $\mathrm{C}_{7}$ and $\left.\mathrm{C}_{8}, J_{\mathrm{C}-\mathrm{F}}=42.8\right), 157.90\left(\mathrm{COCF}_{3}\right.$ in $\left.\mathrm{C}_{\mathrm{c}}, J_{\mathrm{C}-\mathrm{F}}=42.8\right), 114.49\left(\mathrm{COCF}_{3}\right.$, in $\mathrm{C}_{7}$ and $\left.\mathrm{C}_{8}, J_{\mathrm{C}-\mathrm{F}}=283.5\right)$, $114.43\left(\left(\mathrm{COCF}_{3}\right.\right.$, in $\left.\mathrm{C}_{5}, J_{\mathrm{C}-\mathrm{F}}=283.5\right), 83.50(\mathrm{C}-5), 68.41(\mathrm{C}-7, \mathrm{C}-8), 42.17(\mathrm{C}-3 \mathrm{a}, \mathrm{C}-6 \mathrm{a}), 40.01(\mathrm{C}-1, \mathrm{C}-$ 3), 32.74 (C-4, C-6), 29.51 (C-2).

The influence of the solvent showed the uselessness of this esterification effort, because the NMR signals were of no help to find these signals in the NMR spectra in DMSO-d6.

\section{Results and discussions}

TFA is usual used in NMR of alcohols, amines or thiols, whose signals overlap over those of the protons linked to vicinal carbon atoms and made difficult to calculate the coupling constants and to assign the signals to the corrected protons. Due to its acidity, it moved the labile protons $(\mathrm{OH}, \mathrm{NH}, \mathrm{SH})$ to lower field, and also suppress their coupling with the protons linked to the carbon atoms vicinal to oxygen, nitrogen or sulfur. In this paper, NMR spectra of the compounds 1-10 and 13-15 have been done in $\mathrm{CDCl}_{3}$ as solvent and for the compounds 11 and 12 (insoluble in $\mathrm{CDCl}_{3}$ ) in $\mathrm{DMSO}$, followed by addition of TFA in NMR tube. 
This paper started from this idea that for the correct assignment of the signals for the protons $\mathrm{H}_{2}$ and $\mathrm{H}_{5}$ of the endo-azide 1a (Figure 1), TFA was added; but in spectrum began to appear signals at lower field suggesting that here started the esterification reaction of the compound with TFA. The endo-azide 1a was completely esterified in $24 \mathrm{~h}$ to the trifluoroacetate $\mathbf{1 b}$, and this can be observed in both ${ }^{1} \mathrm{H}$ and ${ }^{13} \mathrm{C}$-NMR. In ${ }^{1} \mathrm{H}-\mathrm{NMR}$, the methylene protons of primary alcohol are deshielded from 3.95 to $4.78 \mathrm{ppm}$, respectively 3.92 to $4.68 \mathrm{ppm}$. In ${ }^{13} \mathrm{C}-\mathrm{NMR}$, the chemical shift of carbon atom $\mathrm{C}_{8}$ is deshielded from $60.35 \mathrm{ppm}$ to $66.37 \mathrm{ppm}$, and the vicinal carbon atom $\mathrm{C}_{7}$ is shielded from 51,74 to $47.88 \mathrm{ppm}$. And the signals for trifluoroacetate group are present in the spectrum at: $158.21\left(\mathrm{COCF}_{3}\right)$ and $114.55\left(\mathrm{COCF}_{3}\right)$ ppm. A comparison of ${ }^{1} \mathrm{H}$ and ${ }^{13} \mathrm{C}-\mathrm{NMR}$ spectra of the starting compound $\mathbf{1 a}$ and the esterified compound $\mathbf{1 b}$ is presented in Figure 2.

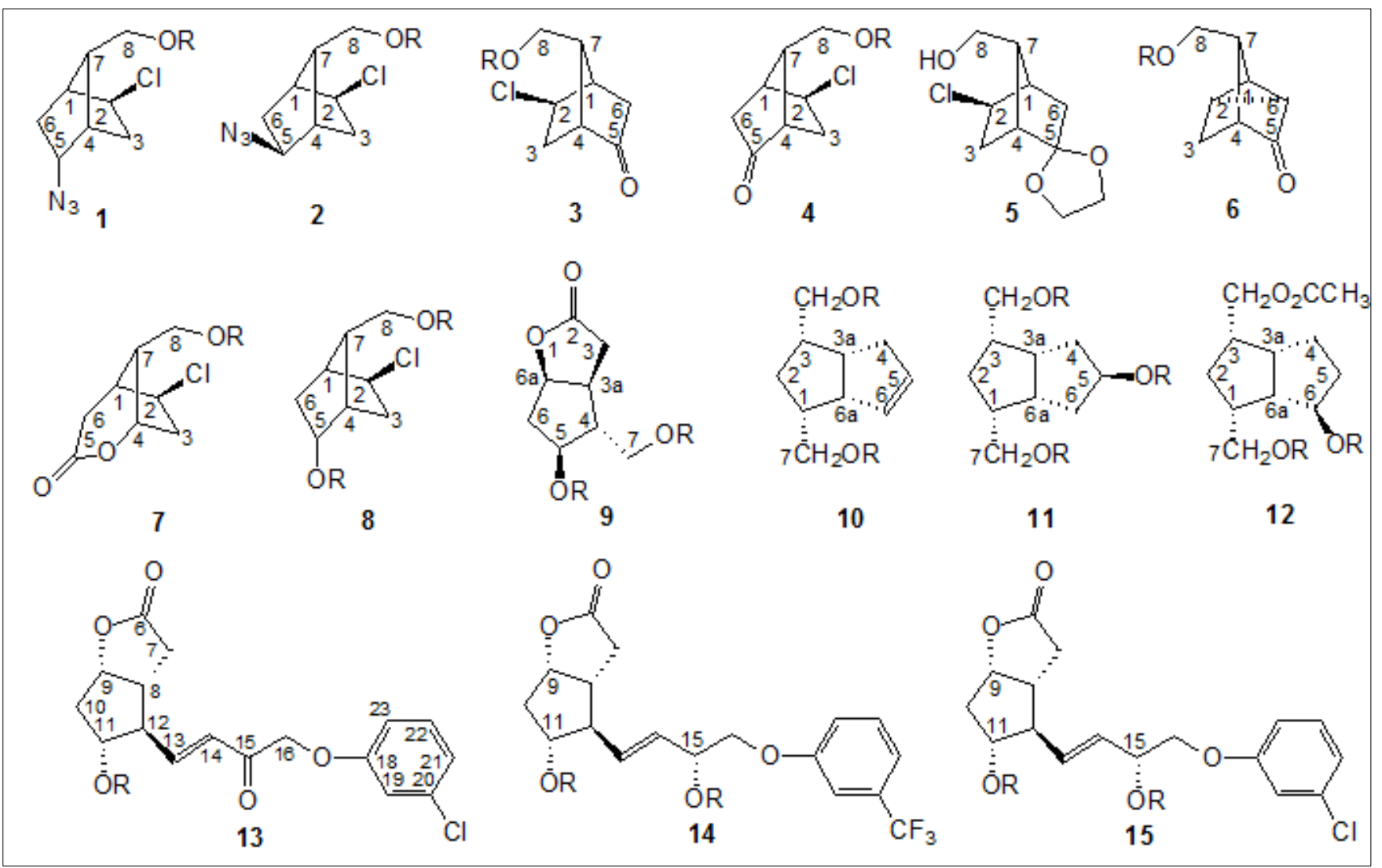

Figure 1. Compounds studied in NMR tube, in $\mathrm{CDCl}_{3}(\mathbf{1 - 1 0}, \mathbf{1 3 - 1 5})$ and DMSO (11 and 12) as solvents without and with TFA; in the paper the compounds with $\mathrm{R}=\mathrm{H}$, are numbered $\mathbf{x a}$ (for example 1a) and the compounds with $\mathrm{R}=\mathrm{CF}_{3} \mathrm{CO}$, are numbered $\mathbf{x b}$ (or $\mathbf{x c}$ ) (for example 1b) 

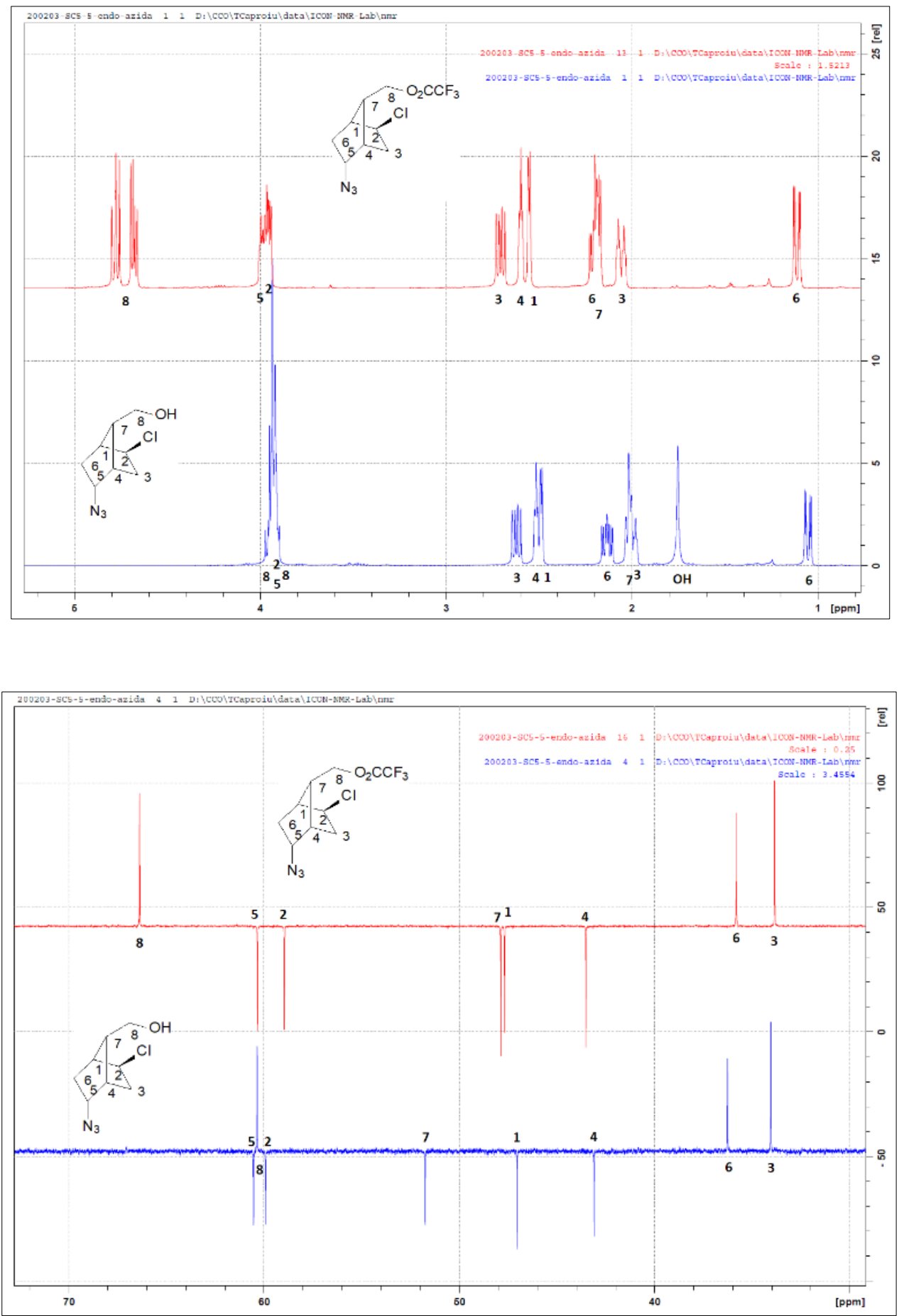

Figure 2. ${ }^{1} \mathrm{H}$ and ${ }^{13} \mathrm{C}$-NMR spectra in $\mathrm{CDCl}_{3}$ of endo-azide 1a and that of the esterified compound $\mathbf{1 b}$, formed in $24 \mathrm{~h}$ by addition of TFA

Starting from this observation, the exo-azide compound 2a was similarly studied by addition of TFA in NMR tube, and the esterification to $\mathbf{2 b}$ was also finished in $24 \mathrm{~h}$ (Figure 3 and Experimental, 2): 

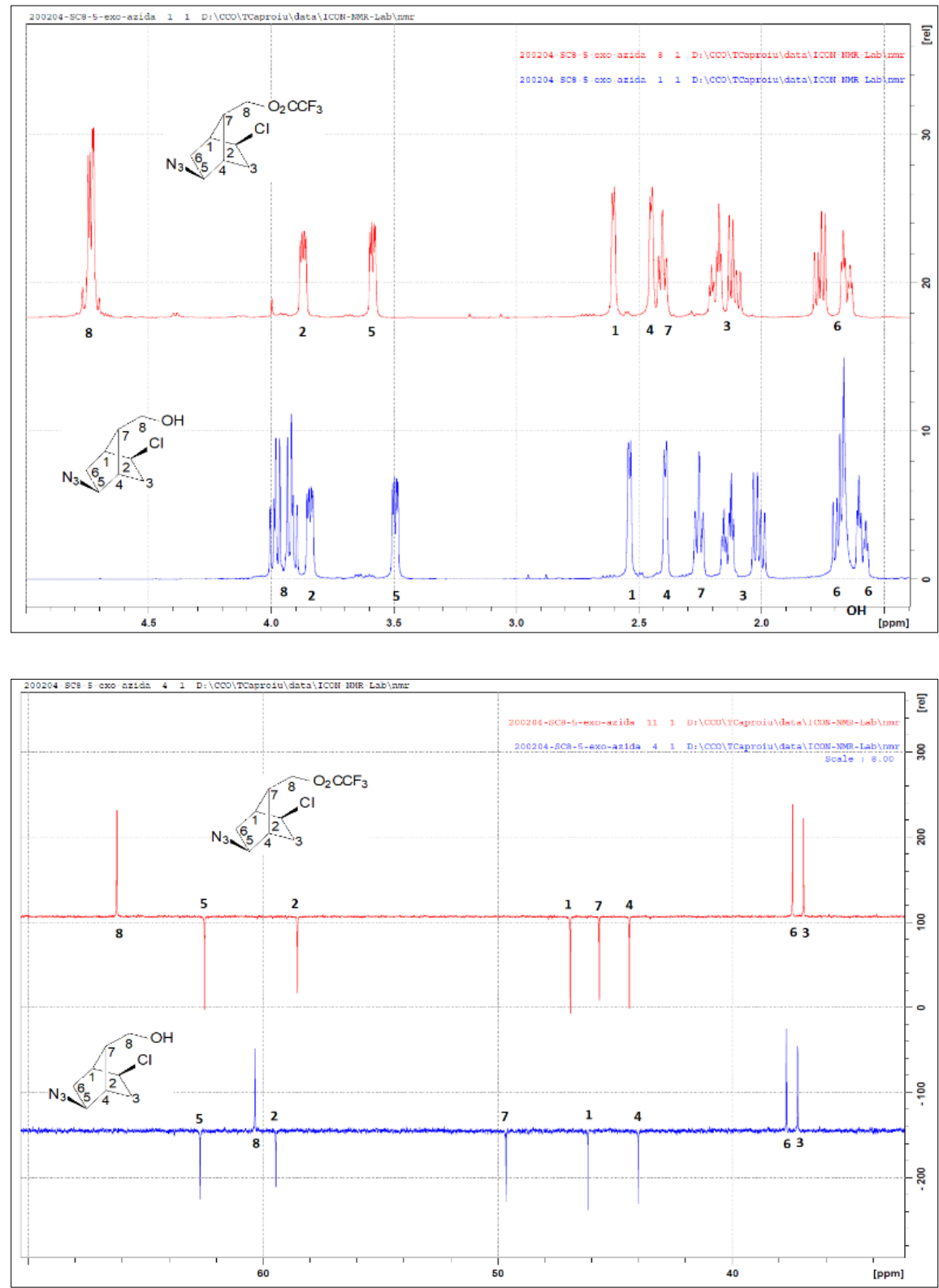

Figure 3. ${ }^{1} \mathrm{H}$ and ${ }^{13} \mathrm{C}$-NMR spectra in $\mathrm{CDCl}_{3}$ of endo-azide $\mathbf{2 a}$ and that of the esterified compound $\mathbf{2 b}$, formed in $24 \mathrm{~h}$ by addition of TFA

Then the study was extended to other compounds with primary alcohol groups (3a-7a), with primary and secondary alcohol groups (8a-12a), a secondary alcohol group alone (13) and a secondary alcohol group in the presence of a secondary allylic alcohol (14-15), in racemic: 5a, 10a-12a, or optically active form: 1a-4a, 6a-9a, 13-15, usually used in our laboratory, presented in Figure 1, which contain:

- a 5-keto group in the bicyclo[2.2.1] heptane skeleton (compounds 3a and 4a) or protected with an ethylene ketal group as in the compound $\mathbf{5 a}$, or in a more constrained structure containing a cyclopropane ring (compound 6a).

- a $\delta$-lactone (compound 7a).

- a primary and a secondary alcohol group in a bicyclo[2.2.1]heptane (compound 8a) or oxabicyclo [3.3.0] octane (ent-Cotey lactone 9a) skeleton.

- a double bond in a bicyclo[3.3.0]octane diol with two primary alcohol groups (compound 10a).

- a bicyclo[3.3.0]octane triol containing two primary alcohol groups and a secondary alcohol group linked to $\mathrm{C}_{5}$ carbon atom (compounds $\left.\mathbf{1 1 a}\right)$ or to $\mathrm{C}_{6}$ carbon atom $(\mathbf{1 2 a}$, with a primary alcohol protected as acetate). 
- an enone $(\mathbf{1 3})$ and two diols $(\mathbf{1 4 , 1 5})$ in prostaglandin intermediate, containing a double bond.

The 5-keto compounds $\mathbf{3 a}$ and $\mathbf{4 a}$ were cleanly esterified with TFA in $24 \mathrm{~h}$ to the compounds $\mathbf{3 b}$ and $4 \mathbf{b}$, and the deshielding of the $\mathrm{H}_{8}$ protons from $\delta=4.05$ to 4.79 and $\delta=3.95 \mathrm{ppm}$ to $4.70 \mathrm{ppm}$ and of the carbon atom $\mathrm{C}_{8}$ from $\delta=59.62 \mathrm{ppm}$ to $64.95 \mathrm{ppm}$ by trifluoro acetyl esterification is observed in NMR spectra, as previously. After 3 days (over weekend), the compound $\mathbf{3 b}$ was obtained pure (Figure 4 and Experimental, 3).
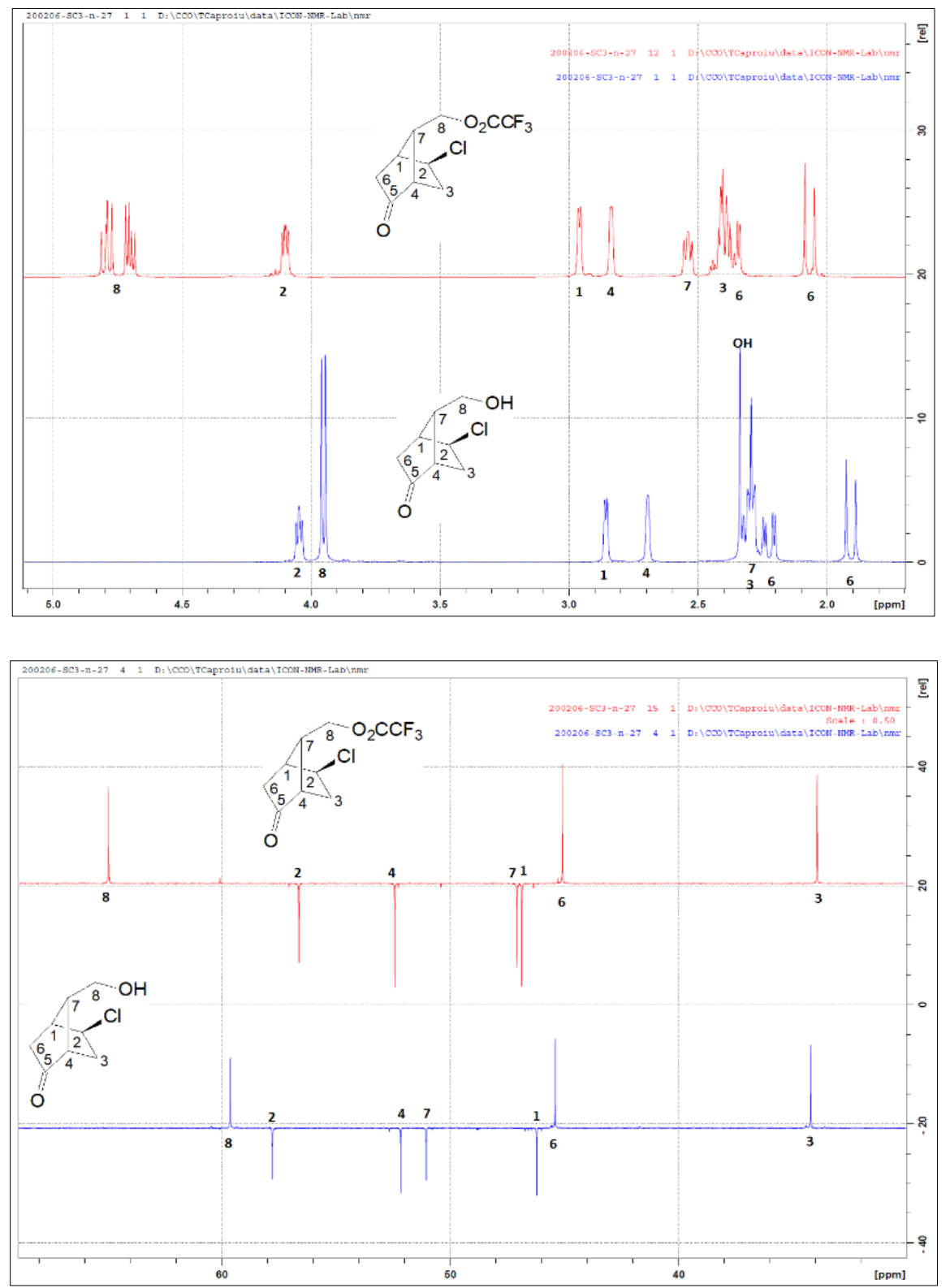

Figure 4. ${ }^{1} \mathrm{H}$ and ${ }^{13} \mathrm{C}$-NMR spectra in $\mathrm{CDCl}_{3}$ of compound $\mathbf{3 a}$ and that of the esterified compound $\mathbf{3 b}$, formed in 3 days by addition of TFA

In the case of the ethylene-ketal protected compound ( \pm )-5a, trifluoroacetic acid esterified the primary alcohol, but in the same time it deprotected the ethylene ketal group giving the compound $\mathbf{3 b}$, as can been observed in ${ }^{13} \mathrm{C}-\mathrm{NMR}$ spectrum where the ketal $\mathrm{C}_{5}$ carbon atom $(\delta=113.84 \mathrm{ppm})$ no more appear in the spectrum, but appear the $\mathrm{C}_{5}$-ketone carbon atom at $216.01 \mathrm{ppm}$ (Figure 5). The ethylene ketal seems to be transformed into ethylene glycol and ethylene glycol mono-trifluoroacetate in a ratio of $1: 1$, as can be observed in ${ }^{1} \mathrm{H}-\mathrm{NMR}$ and ${ }^{13} \mathrm{C}-\mathrm{NMR}$ (Experimental 5, were the signals of ethylene glycol and of the mono-trifluoroacetate are evidenced in red color). 

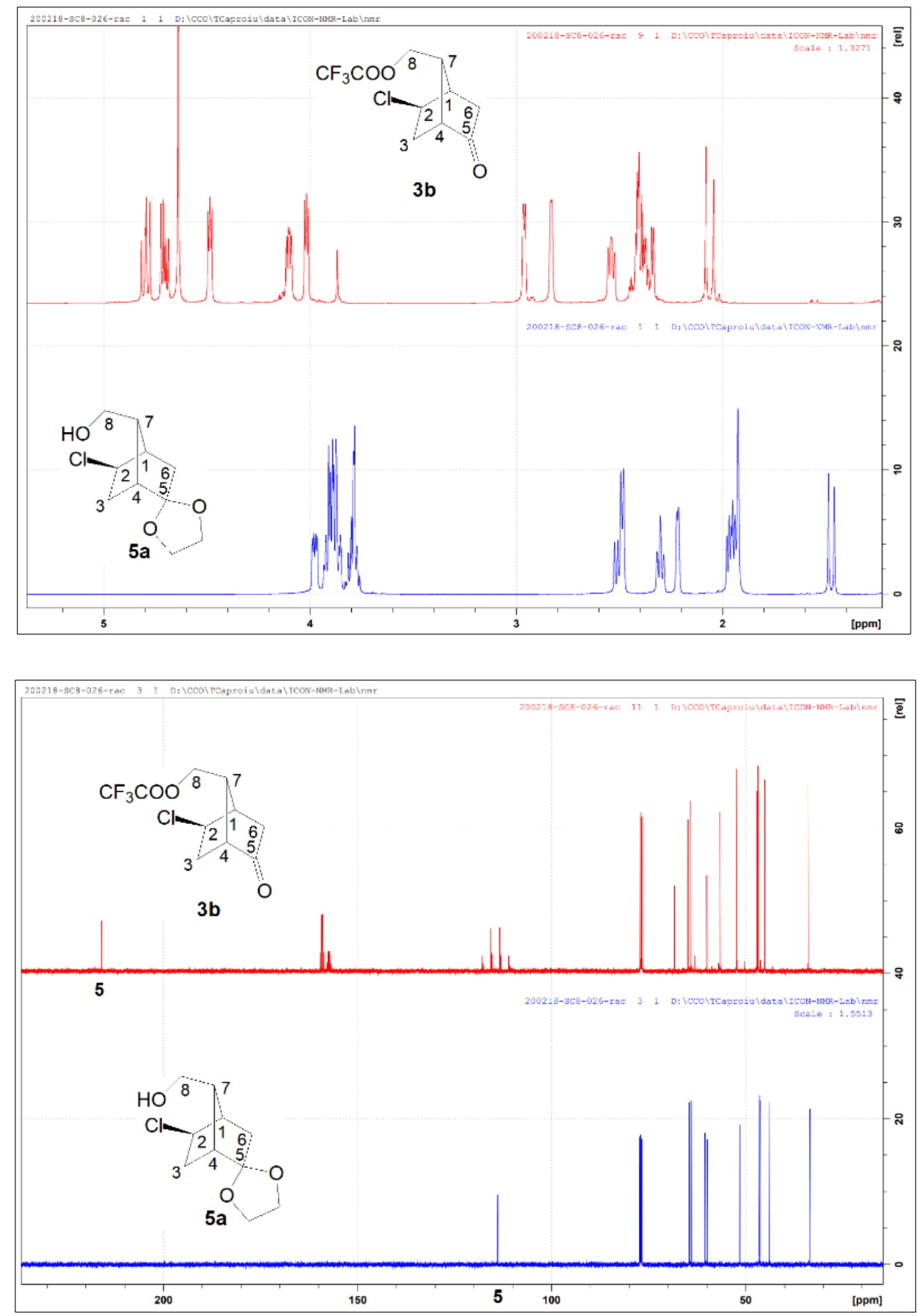

Figure 5. ${ }^{1} \mathrm{H}$ and ${ }^{13} \mathrm{C}$-NMR spectra in $\mathrm{CDCl}_{3}$ of compound $\mathbf{5 a}$ and that of the esterified compound $\mathbf{3 b}$, formed in $24 \mathrm{~h}$ by addition of TFA

The compound 6a, a tricyclic ketone what behaves like an $\alpha, \beta$-unsaturated ketone, was also quantitatively trifluoroacetylated in $48 \mathrm{~h}$ (Experimental, 6).

For the compound $\mathbf{7 a}$, the $\delta$-lactone resists during its quantitative TFA-esterification to the trifluoroacetylated compound $\mathbf{7 b}$ in $24 \mathrm{~h}$ (Experimental, 7).

The diol 8a was esterified with TFA to both the primary alcohol and the secondary alcohol (Figure 6a) and this was observed even after two hours (Figure 6b), but it was not observed that the esterification of the primary alcohol to $\mathbf{8 b}$ proceeded selectively before begins the esterification of the secondary one; in $24 \mathrm{~h}$ the reaction proceeded quantitatively and gave the compound 8c (Experimental 8), which is clearly observed in ${ }^{1} \mathrm{H}$ - and ${ }^{13} \mathrm{C}-\mathrm{NMR}$ (Experimental, 8). In ${ }^{1} \mathrm{H}-\mathrm{NMR}, \mathrm{H}_{5}$ was deshielded from $\delta=4.23$ ppm to $\delta=5.20 \mathrm{ppm}$, and the $\mathrm{H}_{8}$-protons were deshielded from $\delta=3.96$ to $5.20 \mathrm{ppm}$, respectively 3.91 to $4.81 \mathrm{ppm}$. Both trifluoro acetyl groups appear in ${ }^{13} \mathrm{C}-\mathrm{NMR}$ spectra, at 158.24 and $114.54 \mathrm{ppm}$ for $\mathrm{COCF}_{3}$ and $\mathrm{COCF}_{3}$ linked to $\mathrm{OC}_{5}$, respectively 157.80 and $114.41 \mathrm{ppm}$ for $\mathrm{COCF}_{3}$ and $\mathrm{COCF}_{3}$ linked 
to $\mathrm{OC}_{8}$. The signals for $\mathrm{C}_{5}$ and $\mathrm{C}_{8}$ are deshielded from 70.25 , respectively $60.69 \mathrm{ppm}$ to 76.79 , respectively $65.89 \mathrm{ppm}$; even the vicinal carbon atoms to $\mathrm{C}_{5}$ and $\mathrm{C}_{8}$, the carbon atoms $\mathrm{C}_{7}$, respectively $\mathrm{C}_{4}$ and $\mathrm{C}_{6}$ are shielded from $51.38,44.88$ and $39.50 \mathrm{ppm}$ to $47.63,42.95$ and $36.31 \mathrm{ppm}$.

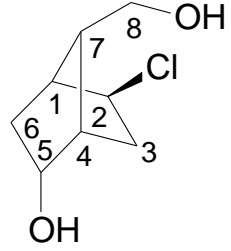

$8 a$

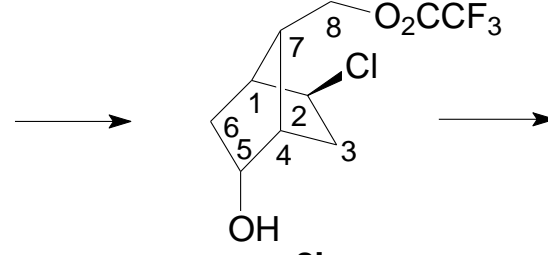

$8 b$

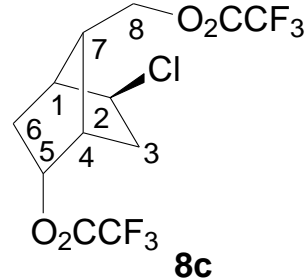

$8 c$

Figure 6a. Trifluoroacetylation of the diol 8a to the diester 8c with TFA in $\mathrm{CDCl}_{3}$

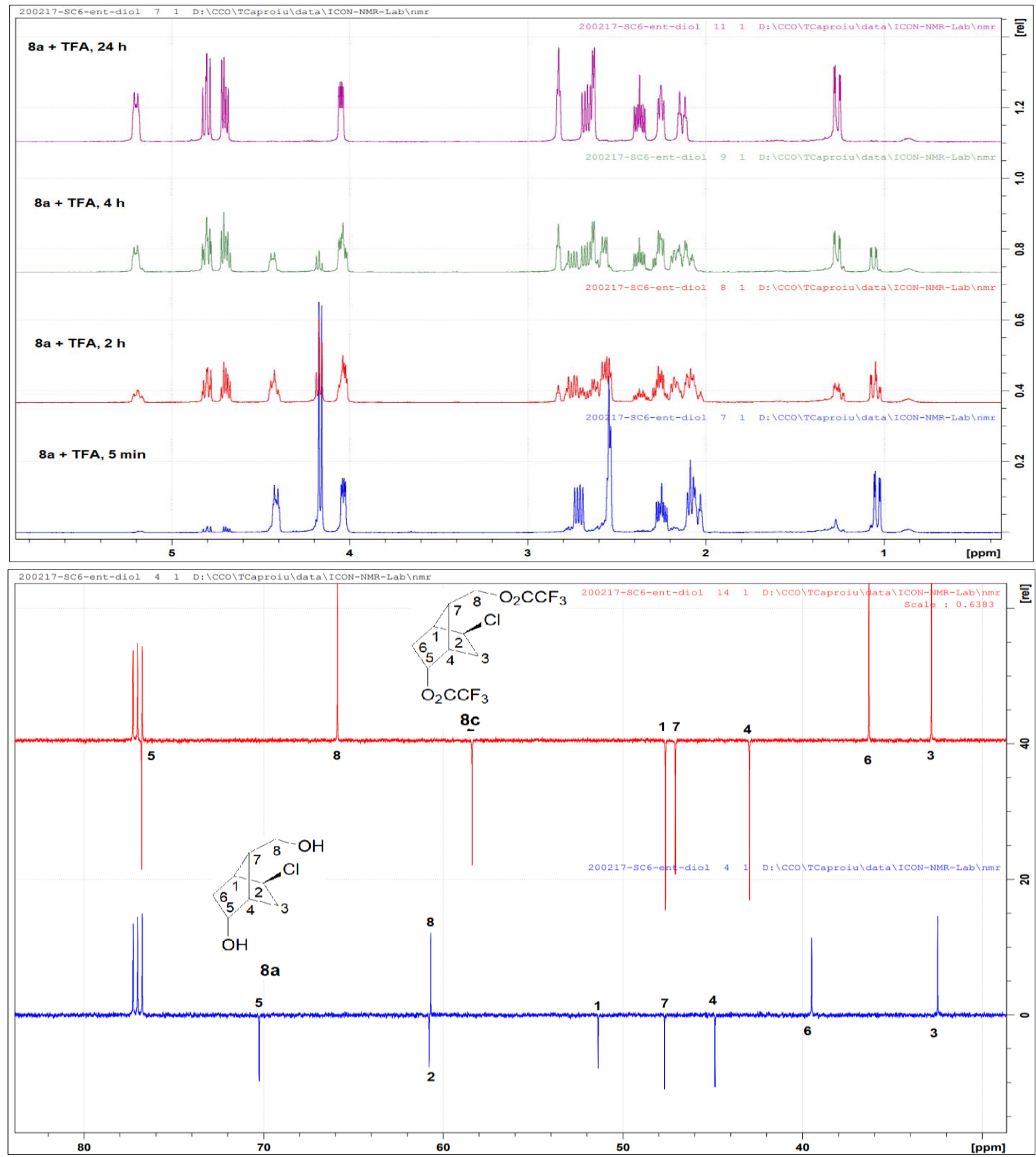

Figure 6b. ${ }^{1} \mathrm{H}$ and ${ }^{13} \mathrm{C}-\mathrm{NMR}$ spectra in $\mathrm{CDCl}_{3}$ of the compound $\mathbf{8 a}$ and that of the esterified compound $\mathbf{8 c}$, formed in $24 \mathrm{~h}$ after addition of TFA 
Corey lactone 9a, with an oxabicyclo[3.3.0]octane fragment, was esterified to both primary and secondary alcohols group, without the observation of the selective trifluoro acetylation of the primary alcohol before begins the trifluoroacetylation of the secondary alcohols (Experimental, 9). In ${ }^{13} \mathrm{C}-\mathrm{NMR}$, both trifluoro acetyl groups appear in NMR spectra, at 157.41 and $114.26 \mathrm{ppm}$ for $\mathrm{COCF}_{3}$ and $\mathrm{COCF}_{3}$ of the primary alcohol ester, respectively 157.10 and $114.61 \mathrm{ppm}$ for $\mathrm{COCF}_{3}$ and $\mathrm{COCF}_{3}$ for the secondary alcohol ester. The deshieldings of the $\mathrm{H}_{5}$ and $\mathrm{H}_{7-}$ protons from 5.27, 4.19 and $3.78 \mathrm{ppm}$ to 5.27, 4.45 and $4.39 \mathrm{ppm}$ and the deshieldings of the corresponding carbon atoms $\mathrm{C}_{5}$ and $\mathrm{C}_{7}$ from 75.65 to $80.60 \mathrm{ppm}$ and 63.73 to $66.57 \mathrm{ppm}$ are clearly observed as previously for $\mathbf{8 a}$ to $8 \mathbf{c}$. The same shieldings of the vicinal carbon atoms to $\mathrm{C}_{7}$ and $\mathrm{C}_{5}, \mathrm{C}_{4}$ and $\mathrm{C}-6$ from 55.02 and $40.81 \mathrm{ppm}$ to 50.61 and $37.59 \mathrm{ppm}$ are also observed, as can been seen in Figure 7.
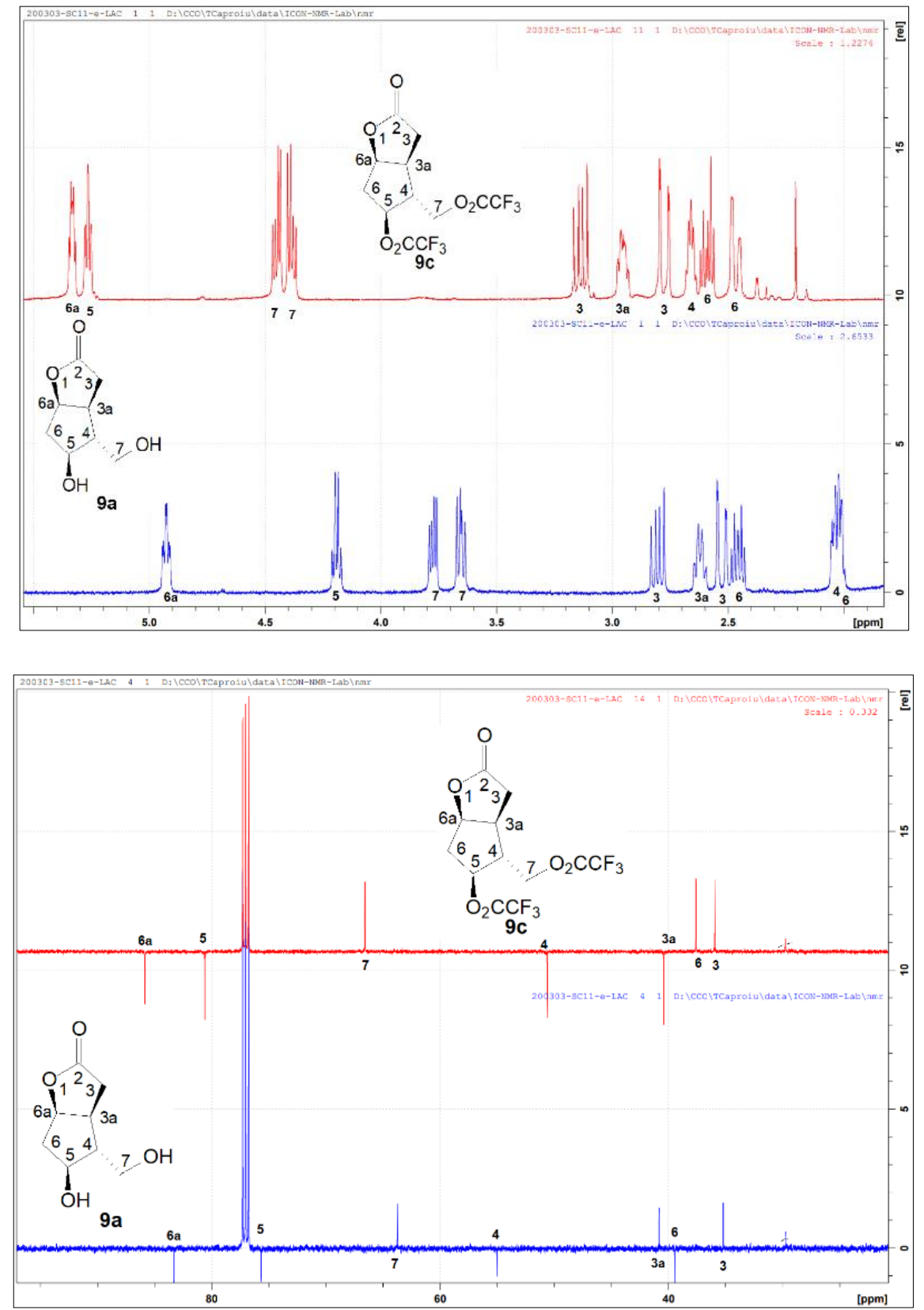

Figure 7. ${ }^{1} \mathrm{H}$ and ${ }^{13} \mathrm{C}$-NMR spectra in $\mathrm{CDCl}_{3}$ of the compound $9 \mathrm{a}$ and that of the esterified compound $9 \mathrm{c}$, formed in $72 \mathrm{~h}$ after addition of TFA 
The double bond of the diol-alkene 10a was not affected during trifluoroacetylation of the primary double bonds to $\mathbf{1 0 b}$. In $4 \mathrm{~h}$, near half esterification of the hydroxyl groups had been done and both hydroxyl groups were esterified after 24 h (Figure 8, Experimental, 10).
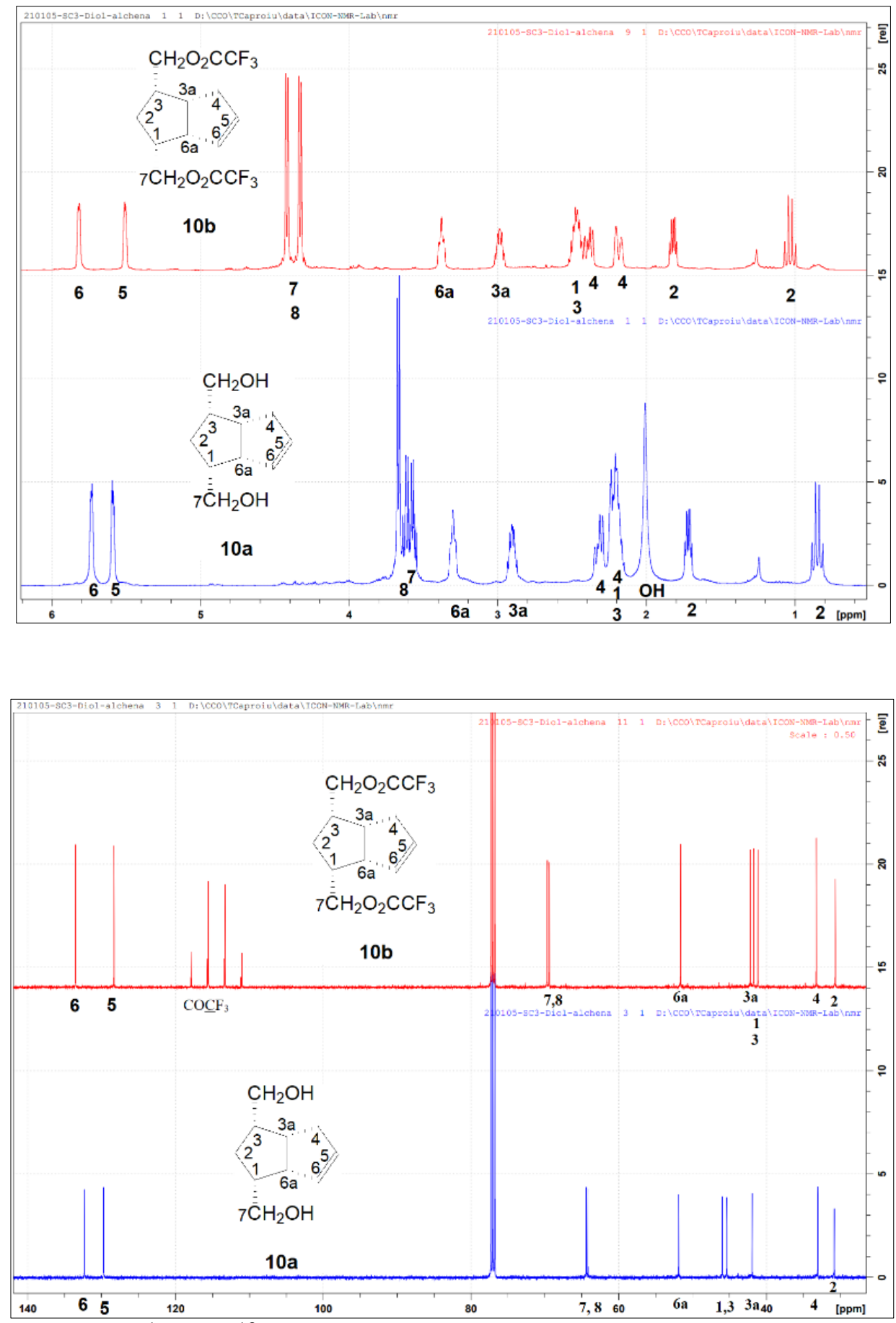

Figure 8. ${ }^{1} \mathrm{H}$ and ${ }^{13} \mathrm{C}$-NMR spectra in $\mathrm{CDCl}_{3}$ of the compound $\mathbf{1 0 a}$ and that of the esterified compound $\mathbf{1 0 b}$, formed in $24 \mathrm{~h}$ after addition of TFA

The enone group of the prostaglandin intermediate 13a remained unmodified during 5 days in the presence of TFA. The deshielding of the proton $\mathrm{H}_{11}$ from 4.39 to the multiplet centered at $5.30 \mathrm{ppm}$ (where it appears together with $\mathrm{H}_{9}$ proton) and of the corresponding carbon atom $\mathrm{C}_{11}$ from $76.55 \mathrm{ppm}$ to $81.73 \mathrm{ppm}$ is also observed (Figure 9). Deshielding of the vicinal carbon atoms $\mathrm{C}_{10}$ and $\mathrm{C}_{12}$ from 40.39 , respectively $56.35 \mathrm{ppm}$ to 37.10 respectively 54.01 is also observed (experimental, 11). 

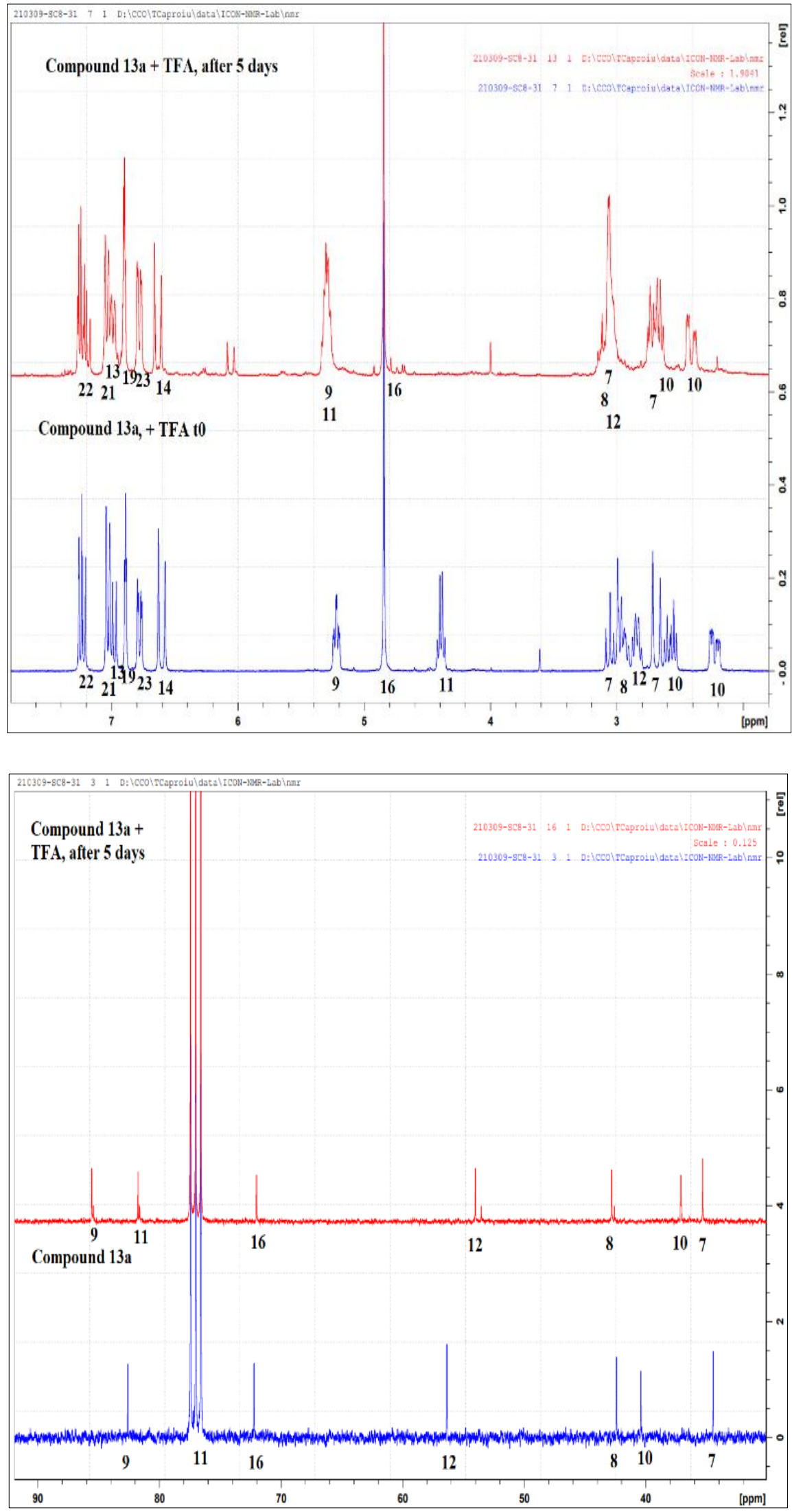

Figure 9. ${ }^{1} \mathrm{H}$ and ${ }^{13} \mathrm{C}-\mathrm{NMR}$ spectra in $\mathrm{CDCl}_{3}$ of the compound 13a and that of the esterified compound 13b, formed after 5 days after addition of TFA 
The prostaglandin diol intermediate 14a, containing a secondary (11-OH) and an allylic secondary (15-OH) alcohol groups was esterified with TFA over weekend (4 days; in $24 \mathrm{~h}$ the bis-trifluoroacetylation did not ended), without concluding that the 15-OH allylic alcohol is selectively esterified than the 9-secondary alcohol (Figure 10 and Experimental 12). The deshielding of the protons $\mathrm{H}_{11}$ and $\mathrm{H}_{15}$ from 4.00 and $4.54 \mathrm{ppm}$ to the multiplets centered at 5.25 and $5.85 \mathrm{ppm}$ and of the corresponding carbon atoms $\mathrm{C}_{11}$ and $\mathrm{C}_{15}$ from 76.52 and $70.50 \mathrm{ppm}$ to 81.74 and $76.39 \mathrm{ppm}$ is clearly observed (Figure 10). The shielding of the vicinal carbon atoms, $\mathrm{C}_{10}, \mathrm{C}_{12}$ and $\mathrm{C}_{16}$ from $39.85,56.35$, respectively 71.81 ppm to $36.66,53.79$, respectively 68.47 is also observed on this diol.
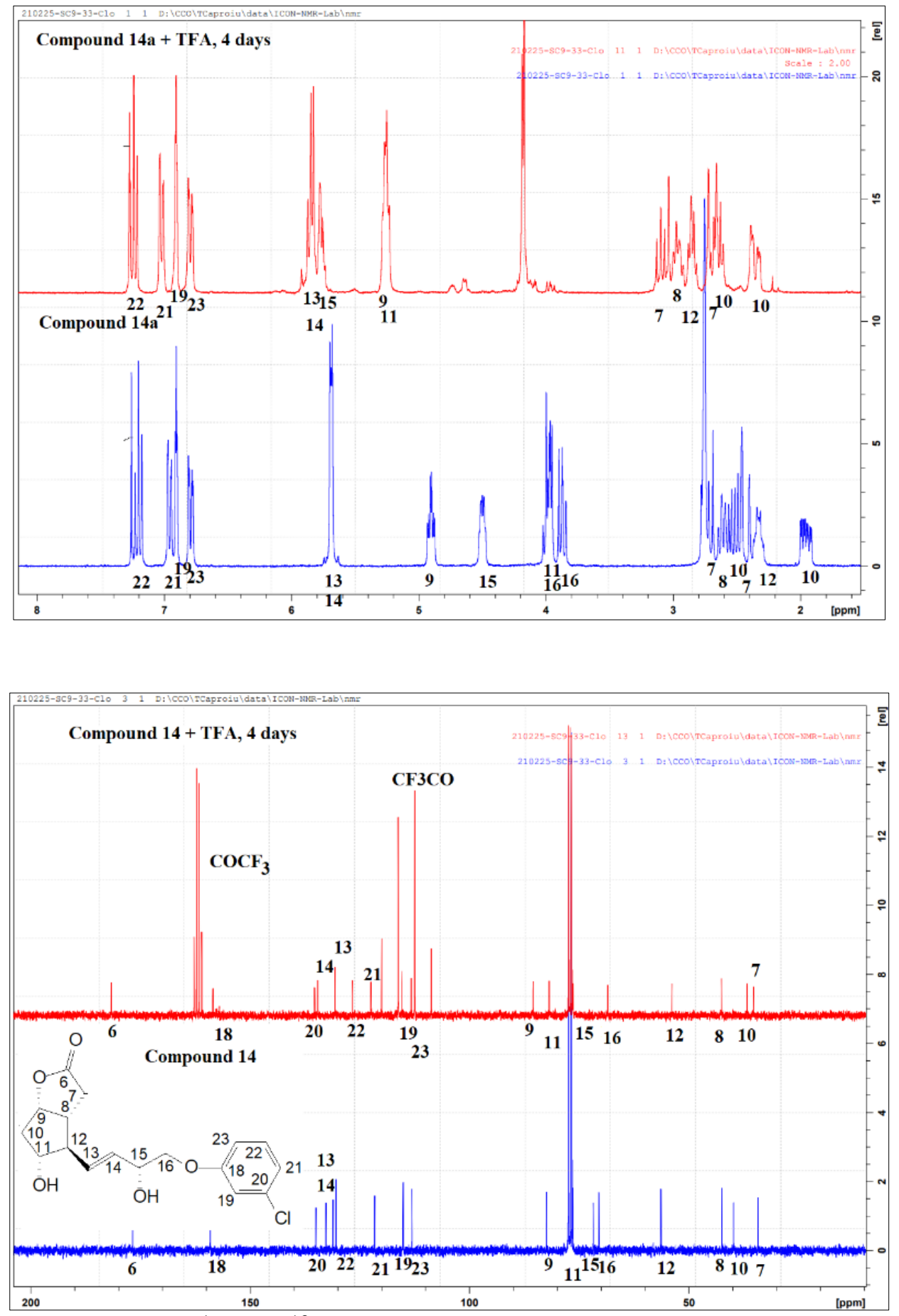

Figure 10. ${ }^{1} \mathrm{H}$ and ${ }^{13} \mathrm{C}-\mathrm{NMR}$ spectra in $\mathrm{CDCl}_{3}$ of the compound 14a and that of the esterified compound 14c, formed in 4 days after addition of TFA 
The same observations are for the diol analog 15a, containing a $m-\mathrm{Cl}$ substituent on the 16-phenoxy group instead of $m$-trifluoromethyl group (Figure 11): the deshielding of the protons $\mathrm{H}_{11}$ and $\mathrm{H}_{15}$ from 4.00 and $4.50 \mathrm{ppm}$ to the multiplets centered at 5.25 and $5.82 \mathrm{ppm}$ and the deshielding of the corresponding carbon atoms $\mathrm{C}_{11}$ and $\mathrm{C}_{15}$ from 76.42 and $70.50 \mathrm{ppm}$ to 81.79 and $76.37 \mathrm{ppm}$ are similar (Figure 11; See als Experimental 13). The shieldings of the vicinal carbon atoms, $\mathrm{C}_{10}, \mathrm{C}_{12}$ and $\mathrm{C}_{16}$ from $39.77,56.32$, respectively $71.74 \mathrm{ppm}$ to $36.67,53.79$, respectively 68.43 are also similar.
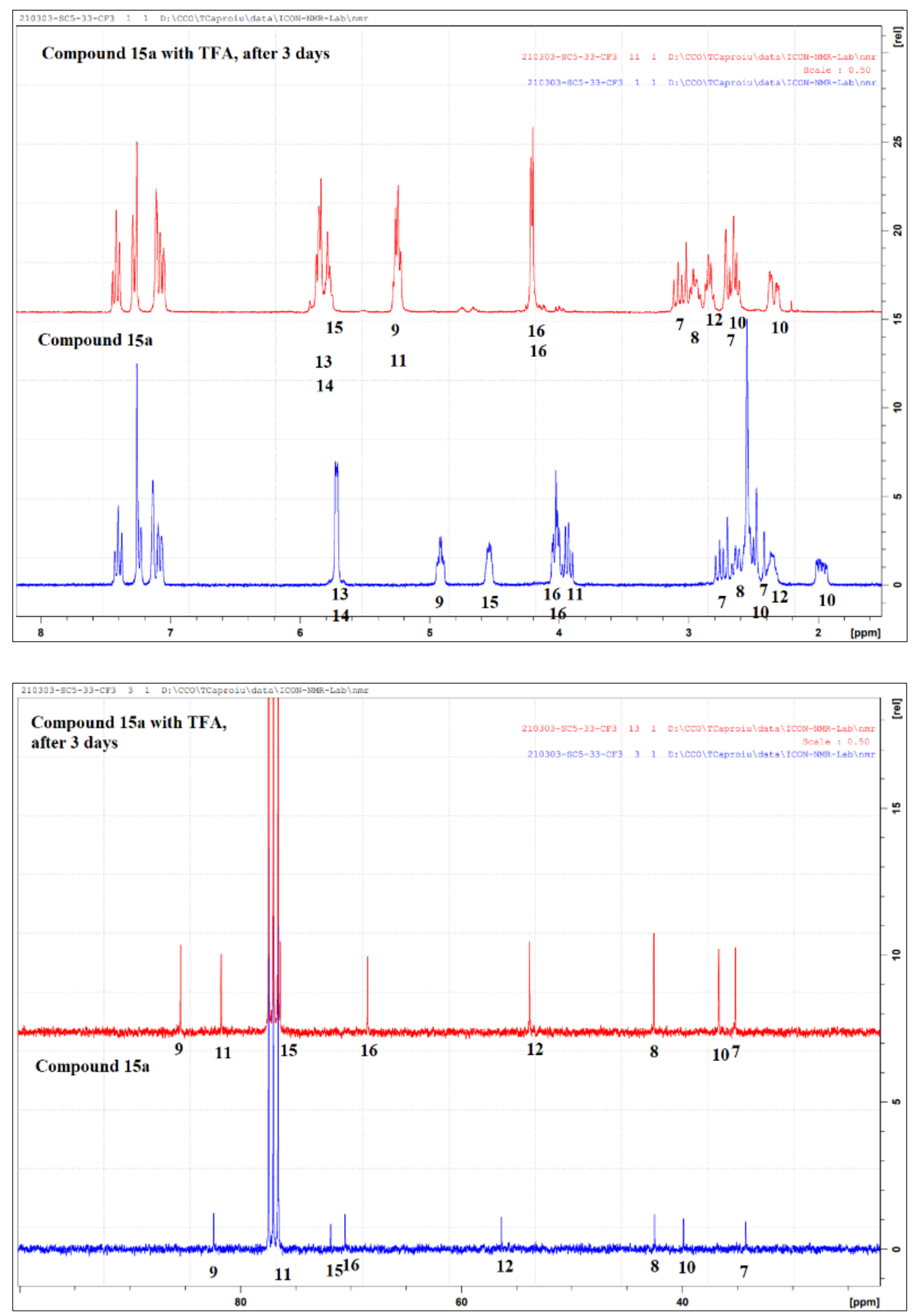

Figure 11. ${ }^{1} \mathrm{H}$ and ${ }^{13} \mathrm{C}-\mathrm{NMR}$ spectra in $\mathrm{CDCl}_{3}$ of the compound $\mathbf{1 5 a}$ and that of the esterified compound 15c, formed in 4 days after addition of TFA

The triols 11 and 12 have low solubility in $\mathrm{CDCl}_{3}$ and NMR spectra were performed in DMSO. In both cases, the esterification with TFA is very slow and the ${ }^{1} \mathrm{H}$ - and ${ }^{13} \mathrm{C}-\mathrm{NMR}$ spectra were not complicated by the formation of the trifluoroacetyl esters. At longer reaction time, the formation of the esters complicated indeed both proton and carbon NMR spectra and the signals couldn't be attributed 
(Experimental, 14, for compound 12a with TFA). The esterification with trifluoroacetic anhydride in TFA proceeded cleanly to the esterified compounds 12c and 11c, and both compounds were fully characterized; unfortunately, because of the different solvents in NMR experiments, the signals of the trifluoroacetylated compounds couldn't be useful to identify them between the compounds formed in DMSO-d6 + TFA (Experimantal 14 and 15). In conclusion, the use of TFA to clarify the NMR spectra of the alcohol compounds in DMSO-d6 at shorter times (even until hours) is beneficial, because the secondary trifluoroacetylated compounds are not formed in amounts to complicate the NMR spectrum.

So, the transformation of the alcohol compounds 1a-10a and 13a-15a into the corresponding trifluoroacetates in $\mathrm{CDCl}_{3}+$ TFA in NMR tubes helped us to correctly attribute or to confirm the NMR signals of some protons and carbon atoms in the molecules, otherwise difficult. Some observations should be mentioned. The primary alcohol groups of the compounds 1a-7a and 10a were quantitatively transformed with TFA into the trifluoroacetylated compounds $\mathbf{1 b}-\mathbf{4 b}, \mathbf{6 b}-\mathbf{7 b}, \mathbf{1 0 b}$ in short time (mainly $24 \mathrm{~h}$ ). Only the ethylene ketal group of the compound 5a was deprotected during the esterification of primary alcohol, giving the trifluoro acetylated compound $\mathbf{3 b}$. The secondary alcohol groups of the compounds 8a and 9a were concomitant esterified with TFA with the primary alcohol groups (to the compounds 8c and 9c), without the observation that the esterification of the secondary alcohols begins after the primary hydroxyl groups were esterified. For compounds $14 \mathbf{a}$ and 15a, the esterification of the secondary 15-allylic alcohols proceeded not selectively against the 11-secondary alcohols; at longer time (3-4 days) both alcohols groups were esterified. It is worth mentioned that the trifluoroacetylation proceeded faster than that in DMSO-d6, where also an additional amount of TFA did not lead to completion of the esterification reaction. The results are to be taken into consideration when TFA is added in NMR tube, because not only the shifting of the couplings of the deuterable protons could help us to simplify the NMR spectrum, but also trifluoro acetylation could help us to more precisely attribute the signals in ${ }^{1} \mathrm{H}$ - and ${ }^{13} \mathrm{C}-\mathrm{NMR}$ spectra to the protons and carbon atoms in the molecule.

\section{Conclusions}

TFA added to the solutions of the compounds $1 \mathbf{a}-10 \mathbf{a}$ and $13 \mathbf{a}-\mathbf{1 5 a}$ in $\mathrm{CDCl}_{3}$ esterified the primary and the secondary alcohols to the corresponding trifluoro acetylated compounds $\mathbf{1 b}-\mathbf{7 b}, \mathbf{8 c}-\mathbf{1 0 c}$ and $\mathbf{1 3 b}$ $\mathbf{1 5 c}$ in 24 to $72 \mathrm{~h}$ in quantitative yield. Ethylene ketal group of the compound 5a was deprotected to the ketone group of the compound $\mathbf{3 b}$, concomitant with the esterification of the primary alcohol. The $\delta$ lactone group of the compound $7 \mathbf{a}$ and $\gamma$-lactone group of the ent-Corey lactone 9a were not opened during esterification of the alcohol groups. The esterification of the secondary 15-allylic alcohols of the compounds 14a and 15a proceeded not selectively against the 11-secondary alcohols, so at longer time (3-4 days) both alcohols groups were esterified.

In DMSO- $\mathrm{d}_{6}$, the esterification of the secondary and primary alcohol groups of the triols 11a and 12a is much slower and didn't went to complete even after 7 days, though the amount of TFA was greater.

In conclusion, TFA added in NMR tube, not only shifts the deuterable protons and simplify the NMR spectrum, but by trifluoroacetylation of the alcohol groups cloud make easier and more precisely attribution of the signals in ${ }^{1} \mathrm{H}$ - and ${ }^{13} \mathrm{C}-\mathrm{NMR}$ spectra to the protons and carbon atoms in the molecule.

Acknowledgments: C.T. and L.P. were supported by the Research Grant NUCLEU: PN 19-41 01 01, 2019 from the Romanian Ministry of Research, Innovation and Digitalization; A.H. gratefully acknowledged the University of Bucharest - UniRem project no. 244 and the contract CNFIS-FDI-20200355 .

\section{References}

1.GILLEN, C. J., KNIPE, A. C., WATTS, W. E. A kinetic study of the mechanism of esterification of 1-arylethanols in trifluoroacetic acid, Tetrahedron Letters 22, 1981, 597 - 600.

2.JOHNSTON, B. H., KNIPE, A. C., WATTS, W. E. A kinetic study of the mechanisms of esterification of alcohols by trifluoroacetic acid. Tetrahedron Lett., 1979, 4225-4226. 
3.SHEN, S., YOU, X. Process for preparation of ethyl trifluoroacetate with trifluoroacetic acid and anhydrous ethanol. 2009, CN 101397249 A 20090401.

4.SMITH, J. B., BYRD, H., O'DONNELL, S. E., DAVIS, W. Hammett Parameter and MolecularModeling Correlations of Substituent Effects on Esterification Kinetics. J. Chem. Educ. 87(8), 2010, 845-847.

5.HU, J., ZHANG, X., YU, F. Process for preparation of ethyl trifluoroacetate. 2017, CN 106699568 A 20170524.

6.C HE, H., XI, Y., WANG, C., ZHONG, Q. Synthetic method of trifluoroacetic acid ethyl ester with strongly acidic cationic exchange resin as catalyst. 2015, CN 104710308 A 20150617.

7.ZHANG, J., LI, G., WANG, Z.; YANG, F. Synthesis of ethyl trifluoroacetate. Hebei Huagong 35(6), 2012, 24-25, 47.

8.AMIET, L., Methyl trifluoroacetate. Eur. Pat. Appl. 1986, EP 206954 A1 19861230.

9.SUN, LANYI; ZHOU, HUI; LI, JUN; GUO, XIAOYAN; LI, QINGSONG. Process and apparatus for production of isopropyl trifluoroacetate. 2009, CN 101362692 A 20090211.

10.GALLAHER, T. N., GAUL, D. A. The esterification of trifluoroacetic acid. J. Chem. Educ. 73(5), 1996, 465-467.

11.MINTER, D. E., VILLARREAL, M. C. The esterification of trifluoroacetic acid. An NMR kinetics experiment. J. Chem. Educ. 62(10), 1985, 911-12.

12.KAVANAGH, P., KNIPE, A. C., WATTS, W. E. Kinetic study of the esterification of arylmethanols in trifluoroacetic acid: mechanistic change consequent upon aryl substituent effects. J. Chem. Soc., Chem. Commun. 20, 1979, 905-6.

13.TĂNASE, I. C., DRĂGHICI, C., CĂPROIU, M. T., SHOVA, S., MATHE, C., COCU, F. G., ENACHE, C., MAGANU, M. New carbocyclic nucleoside analogues with a bicyclo[2.2.1]heptane fragment as sugar moiety; synthesis, X-ray crystallography and anticancer activity, Bioorg. Med. Chem., 22(1), 2014, 513-522.

14.TĂNASE, I. C., DRĂGHICI, C., NEGUT, C., PINTILIE, L. New Constrained Amines in a Bicyclo[2.2.1]Heptane Skeleton, Rev. Chim., 69(9), 2018, 2448-2453.

15.TĂNASE, I. C., DRĂGHICI, C., NEGUT,, C., PINTILIE, L., "Bicyclo[2.2.1]heptane amines protected at the hydroxyl group". RO 133248 A2/2017.

16.TĂNASE, C. I., COCU, F. G., CĂPROIU, M. T., NEAGU, M., MANDA, G. New Bicyclo[2.2.1] heptane Nucleoside Analogues as Antitumor Agents. Rev. Chim., 60(2), 2009, 147-151.

17.TĂNASE, C., COCU, F., CĂPROIU, M. T., DRĂGHICI, C. Key chloroester polyfunctional cyclopentane and oxabicyclo[3.3.0]octane $\gamma$-lactone compounds, obtained by stereo-selective transformations of some $\delta$-lactone intermediates and procedures for their preparation, RO129083B1, 2012.

18.TĂNASE, C., DRĂGHICI, C., HANGANU, A., PINTILIE, L., MAGANU, M., VOLOBUEVA, A., SINEGUBOVA, E., ZARUBAEV, V., NEYTS, J., JOCHMAN, D., SLITA, A. New HSV-1 Anti-Viral 1'-Homocarbocyclic Nucleoside Analogs with an Optically Active Substituted Bicyclo[2.2.1]Heptane Fragment as A Glycoside Moiety, Molecules, 24, 2019, 2446/1-20, doi:10.3390/molecules24132446;

19.TĂNASE, C. I., COCU, F. G., DRĂGHICI, C., CĂPROIU, M. T. 1,2,3,3a,4,6a-Hexahydro-1,3Pentalenedimethanol, mono and bis OH-Protected Derivatives, Useful Intermediates for Fine Organic Synthesis, Rev. Roum.Chim. 53(3), 2008, 195-202.

20.TĂNASE, C., COCU, F., CĂPROIU, M.T., DRĂGHICI, C., SHOVA, S. Hydroboration-Oxidation of $( \pm)-(1 \alpha, 3 \alpha, 3 \mathrm{a} \beta, 6 \mathrm{a} \beta)-1,2,3,3 \mathrm{a}, 4,6 \mathrm{a}-$ Hexahydro-1,3-pentalenedimethanol and Its O-Protected Derivatives: Synthesis of New Compounds Useful for Obtaining (iso)Carbacyclin Analogues and X-ray Analysis of the Products, Molecules, 22(12), 2017, 2032 (1-18).

Manuscript received: 17.03 .2021 